\title{
Polynomial Chaos Expansions for the Stability Analysis of Uncertain Delay Differential Equations*
}

\author{
Rossana Vermiglio ${ }^{\dagger}$
}

\begin{abstract}
In the last few years the polynomial chaos theory of Wiener has been successfully applied to quantify uncertainty in many applications, since it may be a cheap alternative to Monte Carlo simulations. In this paper we introduce linear delay differential equations with uncertain parameters, and we face both the well-posedness of the initial value problem and the stability by means of a suitable abstract reformulation. To quantify the effect of uncertainty on system stability, which is a crucial question in applications, we apply the polynomial chaos expansion to the stability indicator. The proposed numerical method combines the spectral discretization of the infinitesimal generator and the stochastic collocation. Numerical results complete the paper.
\end{abstract}

Key words. uncertain delay differential equations, stability analysis, polynomial chaos expansions

AMS subject classifications. 65Q20, 65P99, 60-08

DOI. $10.1137 / 15 \mathrm{M} 1029618$

1. Introduction. Differential equations involving delay terms play a significant role in modeling real-life phenomena in biology, physics, engineering, medicine, and other sciences. The presence of delay in the models permits a better reproduction of the real dynamics and, as a consequence, a deeper understanding of the phenomena behavior. However, the introduction of past-dependence in the evolution law increases the complexity since, in contrast to ordinary differential equations, delay differential equations are infinite-dimensional dynamical systems. Most authors consider the Banach space $C$ of continuous functions defined on the delay interval equipped with the maximum norm as state space [30, 37, 38, 48, 49]. In fact, as a result of the smoothing effect, the solutions of delay differential equations become continuous, even if other state spaces are used. However, in applications, working in $L^{p}$ spaces (especially $p=2$ ) has some advantages, and there is also a well-developed theory within this framework $[9,6,27,62]$. Nowadays, due to the vast literature on both theoretical and numerical aspects and on applications, it is hard to complete an exhaustive list. The reader interested in a deeper analysis of all the issues can refer to the books $[1,8,19,25,34,45,48,49,50,53,61]$ and to the numerous references therein.

In dynamical systems theory, a first key question is the local stability of equilibria, which, according to the principle of linearized stability, can be inferred from the zero-solution stability of the linearized system. In this context, the understanding of the linear case is crucial,

\footnotetext{
* Received by the editors July 31, 2015; accepted for publication (in revised form) January 24, 2017; published electronically March 23, 2017.

http://www.siam.org/journals/juq/5/M102961.html

Funding: This work was supported by the grant "Numerical analysis of infinite-dimensional and discontinuous differential problems" of the Italian National Scientific Computing Group (2014).

${ }^{\dagger}$ Department of Mathematics, Computer Science and Physics, University of Udine, I-33100 Udine, Italy (rossana.vermiglio@uniud.it).
} 
and the infinitesimal generator of the solution semigroup plays an essential role. In fact the stability is inferred from the position of the rightmost eigenvalues of the infinitesimal generator w.r.t. the imaginary axis. In general, analytical results cannot be achieved, and efficient numerical methods are needed. Recently a pseudospectral differencing method has been proposed within the Banach space $C$ framework [13, 15, 19]. The basis of the approach consists of discretizing the infinitesimal generator to turn the original eigenvalue problem into a matrix eigenvalue problem. The pseudospectral technique guarantees spectral accuracy in the approximation of the eigenvalues. This important feature makes also efficient bifurcation analysis and construction of the stability charts [14]. This so-called IG approach has been extended to other classes of linear differential equations [16, 17] and, more recently, to the nonlinear case too [20]. The idea to discretize the infinitesimal generator, but especially to approximate the solutions of delay differential equations, goes back to the works $[4,5,7,9$, $43,44,51,47,54,56]$. Numerical techniques based upon spline functions within a Hilbert space framework have been proposed, mainly motivated by the interest in control problems. In these cases, the resulting order of approximation for eigenvalues of the infinitesimal generator is fixed [41]. In $[42,46]$ the Legendre-tau approximations were considered and the corresponding approximated characteristic equation was derived, without providing the convergence proof for the eigenvalues. The stability of linear delay systems can be faced by other methodologies, which share the fundamental idea of discretizing the solution operators [11, 18, 19, 32, 39] mainly to investigate the stability of periodic solutions. Finally we recall that the advantage of working with the Hilbert space $L^{2}$ as state space is evident in the computation of Lyapunov exponents [12].

Another important aspect to take into account in practical applications is the specification of initial conditions, model constants, and parameters. In many situations, due to limitations in available experimental data, in measurement or identification of model constants, the input data cannot be exactly specified and are often modeled as random quantities in a suitable probabilistic framework.

In the last few years it has been demonstrated that the polynomial chaos (PC) theory is an important tool to represent random variables and to quantify uncertainty. The PC expansions are based on the original Wiener's theory of homogeneous chaos [63] and on the CameronMartin theorem [22], which states that a random variable with finite second-order moment can be expressed as a convergent series of polynomials in a sequence of random variables. The construction involves an infinite collection of random variables, but, in practice, one reduces the representation to a finite number. The Hermite polynomials, which are the optimum for Gaussian distributions, were the first considered. Other important distributions with the specific family of orthogonal polynomials are introduced in [57], whereas the convergence results are given in [35].

The theory of Fourier series can be applied within this context. Provided that the coefficients of PC representations are known, the statistical moments of all orders and the probability distributions can be obtained at low computational cost. The goal of the uncertainty quantification is to determine the statistical moments of the model solution, subject to parametric uncertainties. In this context, the PC expansions, coupled with efficient numerical methods, provide a convenient framework for the forward propagation of the uncertainties. Whenever the number of uncertain parameters is small and there is a smooth dependency on 
them, these techniques exhibit fast and ideally exponential convergence rate, offering efficient computational strategies. Within this context they are a cheap alternative to Monte Carlo simulations. For a detailed introduction to PC expansions, an overview of the latest methods and various applications, the reader is referred to the books $[52,59]$.

The aim of this paper is to analyze the robustness of linear delay equations, quantifying the effect of data uncertainty on the stability indicator, i.e., the rightmost eigenvalues of the infinitesimal generator. The PC theory provides the basis for the novel definition of delay differential equations with uncertain parameters, here called uncertain delay differential equations. First, after reformulating such equations as abstract delay differential equations on a suitable Hilbert space, we will investigate the well-posedness of the associated initial value problem. Moreover, we will show that the norm-stability of the zero solution can be analyzed through the spectrum of the infinitesimal generator, since this is not the general situation for abstract delay differential equations. Then we will apply the IG approach, and we will solve the resulting random eigenvalue problem by means of the stochastic collocation (SC) method, i.e., the PC expansion coupled with the collocation on a selected set of nodes. The SC method requires only deterministic solvers, and it permits us to obtain a selection of statistical indicators. The stochastic Galerkin (SG) method, i.e., the PC representation coupled with the Galerkin projection, was considered to solve symmetric random eigenvalue problems in [36]. We remark that both approaches were used successfully for time-integration of different classes of differential equations [3, 52, 57, 58, 59].

The paper is organized to be self-contained. In section 2 , bearing in mind a Hilbert space setting for uncertain delay differential equations and Fubini's theorem, we will briefly introduce the basic notation, the fundamental results, and the discretization approach of the infinitesimal generator for deterministic linear delay differential equations in $L^{2}$ spaces. Although part of the material is related to known results, it still needs to be presented to introduce the fundamental ideas clearly. Section 2 ends with the IG approach in $L^{2}$ spaces and the convergence proof for the eigenvalues. After a brief introduction to PC expansions in section 3, we will define uncertain delay differential equations in section 4 , which is the main part of this article. By means of a suitable abstract reformulation, we will study the well-posedness of the initial value problem and the mean-square stability of the zero solution through the semigroup theory. In section 4.3 we will derive a random eigenvalue problem and solve it by combining the IG approach and the SC method. In section 5 we will give some numerical results and applications before drawing conclusions in section 6 .

2. Linear delay differential equations. In this section we first introduce the notation and summarize some basic theoretical results, for which the reader is referred to [6, 9, 27, 62].

Throughout the whole paper we denote the independent variable time by $t \in \mathbb{R}_{+}$and the dimension of the system by $d \geq 1$. The space $\mathbb{R}^{d}$ is equipped with the euclidean norm $|x|_{2}$, $x=\left(x_{1}, \ldots, x_{d}\right)^{T} \in \mathbb{R}^{d} \cdot\|\cdot\|$ indicates the operator norm.

Let $\tau>0$ be the maximum delay. We consider a linear autonomous delay differential equation (DDE) of the form

$$
\frac{d}{d t} y(t)=\mathrm{B} y(t)+L y_{t}, t \geq 0,
$$


where $\mathrm{B} \in \mathbb{R}^{d \times d}$, the function

$$
y_{t}(\theta):=y(t+\theta), \theta \in[-\tau, 0],
$$

represents the history segment for any $t \geq 0$ lying on the history space $H:=L^{2}\left(-\tau, 0 ; \mathbb{R}^{d}\right)$, and $L: W^{1,2}\left(-\tau, 0 ; \mathbb{R}^{d}\right) \rightarrow \mathbb{R}^{d}$ is a linear and bounded operator, called the delay operator. The space $H$ is a Hilbert space, equipped with the inner product

$$
\langle\varphi, \psi\rangle_{H}=\int_{-\tau}^{0} \varphi(\theta)^{T} \psi(\theta) d \theta, \varphi, \psi \in H
$$

and the induced norm $\|\varphi\|_{H}=\sqrt{\langle\varphi, \varphi\rangle_{H}}$. The choice of considering delay operators defined on $W:=W^{1,2}\left(-\tau, 0 ; \mathbb{R}^{d}\right)$, which was proposed by several authors, e.g., [6, 56], allows us to represent both discrete and distributed delays, i.e., linear operators $L$ of the form

$$
L \varphi=\sum_{k=1}^{r} \mathrm{~B}_{k} \varphi\left(-\tau_{k}\right)+\int_{-\tau}^{0} \mathrm{C}(\theta) \varphi(\theta) d \theta, t \geq 0, \varphi \in W,
$$

where $0<\tau_{1}<\cdots<\tau_{r}:=\tau$ are $r$ distinct delays, $\mathrm{B}_{k} \in \mathbb{R}^{d \times d}$ for $k=1, \ldots, r$, and $\mathrm{C}(\cdot) \in$ $L^{2}\left(-\tau, 0 ; \mathbb{R}^{d \times d}\right)$. In fact $W$ is continuously embedded in the space of continuous functions $C:=C\left([-\tau, 0], \mathbb{R}^{d}\right)$, and the bounded linear operator on $C$ given by the Riemann-Stieltjes integral

$$
L \varphi=\int_{-\tau}^{0} d \eta(\theta) \varphi(\theta)
$$

where $\eta:[-\tau, 0] \rightarrow \mathbb{R}^{d \times d}$ is a bounded variation matrix, defines a bounded linear operator also on $W$. We remark that the "distributed delay" contribution in (3) defines a linear and bounded operator on $H$ [6, Example 3.27]. Hereafter we consider DDE (1) with delay operator $L$ represented by (4).

For any $x \in \mathbb{R}^{d}$ and $\varphi \in H$, the initial value problem (IVP) for (1) reads as

$$
\left\{\begin{aligned}
\frac{d}{d t} y(t) & =\mathrm{B} y(t)+L y_{t}, t \geq 0 \\
y(0) & =x \\
y_{0} & =\varphi
\end{aligned}\right.
$$

In the literature a distinction is often made between classical and mild solutions, depending on whether one refers to (1) or to an integral reformulation.

Definition 2.1. We say that $y(\cdot)=y(\cdot ; x, \varphi)$ is a classical solution of $(5)$ on $[-\tau,+\infty)$ if $y \in C\left([-\tau,+\infty), \mathbb{R}^{d}\right) \cap C^{1}\left([0,+\infty), \mathbb{R}^{d}\right), y_{t} \in W$, for all $t \geq 0$, and $y$ satisfies $(5)$.

Definition 2.2. We say that $y(\cdot)=y(\cdot ; x, \varphi)$ is a mild solution of $(5)$ on $[-\tau,+\infty)$ if $\int_{0}^{t} y_{s} d s \in W, t \geq 0$, and $y$ satisfies the integral equation

$$
y(t)= \begin{cases}x+B \int_{0}^{t} y(s) d s+L \int_{0}^{t} y_{s} d s, & t \geq 0, \\ \varphi, & \text { a.e. }-\tau \leq t<0 .\end{cases}
$$


For any $x \in \mathbb{R}^{d}$ and $\varphi \in H$, the IVP (5) has a unique (classical or mild) solution. It is worthwhile stressing that in case of a classical solution we should assume that the initial data $\varphi \in W$ and $\varphi(0)=x$.

As commonly used in case of DDEs on $L^{p}$-spaces, the product space $E:=\mathbb{R}^{d} \times H$ is the state space, which is a separable Hilbert space equipped with the inner product defined as

$$
\langle(x, \varphi),(z, \psi)\rangle_{E}=x^{T} z+\langle\varphi, \psi\rangle_{H},(x, \varphi),(z, \psi) \in E,
$$

and the induced norm

$$
\|(x, \varphi)\|_{E}=\sqrt{|x|_{2}^{2}+\|\varphi\|_{H}^{2}},(x, \varphi) \in E .
$$

For a given solution $y$ of (5), the state at time $t \geq 0$ is defined as

$$
u(t):=\left(y(t), y_{t}\right)
$$

This setting is sometimes referred as extended state space and extended state to distinguish them, respectively, from the standard state space $C$ and the standard state $y_{t} \in C$.

The solution operator $T(t): E \rightarrow E$ is the linear and bounded operator, which associates the state $\left(y(t), y_{t}\right) \in E$ at time $t$ with the initial state $(x, \varphi) \in E$, i.e.,

$$
T(t)(x, \varphi)=\left(y(t), y_{t}\right), t \geq 0 .
$$

The family $\{T(t)\}_{t \geq 0}$, called SO-semigroup, forms a $C_{0}$-semigroup, which is eventually compact [9]. The associated infinitesimal generator $A: D(A) \subseteq E \rightarrow E$ is the linear unbounded operator given by

$$
\left\{\begin{array}{l}
A(x, \varphi)=\left(\mathrm{B} x+L \varphi, \frac{d}{d \theta} \varphi\right) \\
D(A)=\left\{(x, \varphi) \in \mathbb{R}^{d} \times W: \varphi(0)=x\right\}
\end{array}\right.
$$

where $\frac{d}{d \theta}$ is the weak-derivative.

The abstract Cauchy problem (ACP) associated with $A$ reads as

$$
\left\{\begin{array}{l}
u^{\prime}(t)=A u(t), \quad t \geq 0 \\
u(0)=(x, \varphi)
\end{array}\right.
$$

The notions of classical and mild solutions can also be introduced for (9). It can be proved that the IVP (5) and the ACP (9) are equivalent in the sense that any classical solution $t \mapsto y(t)$ of (5) defines a classical solution $t \mapsto u(t)=\left(y(t), y_{t}\right)$ of (9) and vice versa. This result also holds for mild solutions of (9), which are continuous functions such that $\int_{0}^{t} u(s) d s \in D(A)$ and $u(t)=(x, \varphi)+A \int_{0}^{t} u(s) d s, t \geq 0$.

Once the well-posedness of the IVP (5) has been established, we can focus our attention on the stability of the zero solution of (1), which is a special but significant problem. In fact, according to the principle of linearized stability, the stability properties of the steady state solution $\bar{y}(t):=\bar{y}$ of the nonlinear autonomous DDE

$$
\frac{d}{d t} y(t)=F\left(y(t), y_{t}\right), \quad t \geq 0,
$$


where $F: E \rightarrow \mathbb{R}^{d}$ is a continuous differentiable map, can be linked to the stability of the zero solution of the linearized system at $\bar{y}$, i.e.,

$$
\frac{d}{d t} y(t)=D F_{1}(\bar{y}, \bar{y}) y(t)+D F_{2}(\bar{y}, \bar{y}) y_{t}, \quad t \geq 0,
$$

where $D F_{1}(x, \varphi), D F_{2}(x, \varphi)$ are the derivatives of $F$ at $(x, \varphi)$ w.r.t. $x$ and $\varphi$, respectively.

The spectral theory provides a powerful tool to study the long-time behavior of semigroups $[24,31]$. To this aim we assume that all the spaces and operators involved have been complexified [30]. The eventually compactness of the SO-semigroup allows us to prove that its growth bound, i.e.,

$$
\omega_{0}(T):=\inf \left\{\omega \in \mathbb{R}: \exists M>0 \text { such that }\|T(t)\| \leq M e^{\omega t}, t \geq 0\right\},
$$

coincides with the spectral abscissa, i.e.,

$$
s(A):=\sup \{\mathcal{R} e(\lambda): \lambda \in \sigma(A)\} .
$$

This result is fundamental, since it states that the "best possible" exponential growth of classical and mild solutions can be determined without constructing the SO-semigroup. The spectrum $\sigma(A)$ of the infinitesimal generator $A$ only contains eigenvalues. Hence, the zero solution of (1) is asymptotically stable if and only if all the (infinitely many) eigenvalues $\lambda$ of $A$ lie in the left-half complex plane. By observing that for the eigenvector $(x, \psi)$ associated to the eigenvalue $\lambda$ it results in $\psi(\cdot ; x, \lambda)=x e^{\lambda \cdot}$, it is possible to derive the characteristic equation

$$
\operatorname{det}\left(\lambda I_{d}-\mathrm{B}-L e^{\lambda \cdot}\right)=0
$$

whose solutions, called the characteristic roots, coincide with the eigenvalues of $A$. Finally we recall that any right-half plane contains only finitely many characteristic roots. By looking at (13) we realize that it does not really matter which state space is chosen, since the characteristic equation remains unchanged.

The stability analysis requires suitable numerical methods. Even if the characteristic roots can be computed by solving (13), it is well known that this approach may lead to illconditioning. In $[13,19]$ the infinite-dimensional eigenvalue problem for $A$ on the state space $C$ is turned into a matrix eigenvalue problem by means of the pseudospectral discretization. Below we will briefly present the setting of the so-called IG approach for the state space $E$, and we will give the proof of convergence of the eigenvalues. Having to work on the domain $D(A)$, we restrict ourselves to $\varphi \in W$.

For a given integer $N$, the IG approach consists of substituting the exact function $\varphi \in W$ (infinite dimension) with its interpolating polynomial $\varphi_{N} \in W_{N}$ (finite dimension) at the $N+1$ distinct nodes in $[-\tau, 0]$,

$$
\Theta_{N}:=\left\{-\tau \leq \theta_{N}<\cdots<\theta_{1}<\theta_{0}=0\right\},
$$

and by applying the infinitesimal generator $A$ to $\left(\varphi_{N}(0), \varphi_{N}\right) \in \mathbb{R}^{d} \times W_{N}$, i.e.,

$$
A_{N}\left(\varphi_{N}(0), \varphi_{N}\right)=\left(\mathrm{B} \varphi_{N}(0)+L \varphi_{N}, \frac{d}{d \theta} \varphi_{N}\right) \text {. }
$$


We remark that the choice $\theta_{0}=0$ is motivated by the domain condition. The convergence of the eigenvalues is achieved through the same strategy in $[13,19]$, which compares the characteristic equation (13) to the discrete one by means of Rouché's theorem. The latter is given by

$$
\operatorname{det}\left(\lambda I_{d}-\mathrm{B}-L\left(\psi_{N}(\cdot ; \lambda, x)\right)\right)=0,
$$

where $\psi_{N}(\cdot ; \lambda, x)$ is the $N$-degree $\mathbb{C}^{d}$-valued collocation polynomial relevant to the nodes $\theta_{i}, i=1, \ldots, N$, for the problem

$$
\left\{\begin{array}{l}
\psi^{\prime}(\theta)=\lambda \psi(\theta), \theta \in[-\tau, 0] \\
\psi(0)=x
\end{array}\right.
$$

whose solution is $\psi(\theta ; \lambda, x)=e^{\lambda \theta} x, \theta \in[-\tau, 0]$. In this context the existence and uniqueness of the polynomial $\psi_{N}$ and an estimate of the error $e_{N}=\psi_{N}-\psi$ are key questions.

Lemma 2.3. Assume that $\theta_{i}, i=1, \ldots, N$, are the zeros of the $N$-degree Legendre polynomial in $[-\tau, 0]$. Let $\lambda^{*} \in \mathbb{C}$ and $\rho>0$. Then there exists $N_{0}:=N_{0}(\rho)$ such that, for $N \geq N_{0}$, and $\lambda$ s.t. $\left|\lambda-\lambda^{*}\right| \leq \rho$, and $x \in \mathbb{C}^{d}$, the collocation polynomial $\psi_{N}$ for (16) relevant to $\theta_{i}, i, \ldots, N$, exists and it is unique. Moreover, we obtain the bound

$$
\left\|\psi_{N}(\cdot ; \lambda, x)-\psi(\cdot ; \lambda, x)\right\|_{H} \leq \frac{C_{1}}{\sqrt{N}}\left(\frac{C_{2}}{N}\right)^{N}|x|_{2},
$$

where $C_{1}:=C_{1}\left(\lambda^{*}, \rho\right), C_{2}:=C_{2}\left(\lambda^{*}, \rho\right)$ are constants independent of $N$.

Proof. By introducing the linear and bounded Volterra operator $\mathcal{K}: H \rightarrow H$

$$
(\mathcal{K} \varphi)(\theta)=\int_{0}^{\theta} \varphi(\theta) d \theta, \varphi \in H, \theta \in[-\tau, 0] .
$$

We can express $\psi, \psi_{N}$ as follows:

$$
\begin{aligned}
& \psi=x+\lambda \mathcal{K} \psi \\
& \psi_{N}=x+\lambda \mathcal{K} \mathcal{L}_{N-1} \psi_{N},
\end{aligned}
$$

where $\mathcal{L}_{N-1}$ is the interpolation operator relevant to the nodes $\theta_{i}, i=1, \ldots, N$. As in [19, Proposition 5.1], we easily obtain that the error $e_{N}=\psi-\psi_{N}$ can be written as $e_{N}=\lambda \mathcal{K} \widehat{e}_{N}$, where $\widehat{e}_{N}$ is the solution of

$$
\left(I_{H}-\lambda \mathcal{L}_{N-1}\right) \mathcal{K} \widehat{e}_{N}=r_{N}
$$

and $r_{N}:=\mathcal{L}_{N-1} \psi-\psi$ is the consistency error. Moreover $\left(I_{H}-\lambda \mathcal{L}_{N-1} \mathcal{K}\right)=\left(I_{H}-\lambda \mathcal{K}\right)+$ $\lambda\left(I_{H}-\mathcal{L}_{N-1}\right) \mathcal{K}$. The operator $I_{H}-\lambda \mathcal{K}$ is invertible with bounded inverse, whereas the norm of operator $\left(I_{H}-\lambda \mathcal{L}_{N-1}\right) \mathcal{K}$ goes to zero as $N$ goes to infinity according to the Erdős-Turán theorem [33]. Therefore there exists $N_{0}:=N_{0}(\rho)$ such that, for $N \geq N_{0}$ and $\left|\lambda-\lambda^{*}\right| \leq \rho$, $\left\|\left(I_{H}-\lambda \mathcal{L}_{N-1}\right) \mathcal{K}\right\|_{H} \leq \frac{1}{2}$. By means of the Banach perturbation lemma, we can conclude that $\left(I_{H}-\mathcal{L}_{N-1}\right) \mathcal{K}$ is invertible and its inverse is uniformly bounded. Therefore, we can find a constant $C_{0}:=C_{0}\left(\lambda^{*}, \rho\right)$ such that $\left\|\lambda \mathcal{K}\left(\left(I_{H}-\mathcal{L}_{N}\right) \mathcal{K}\right)^{-1}\right\|_{H} \leq C_{0}$ for $N \geq N_{0}$ and 
$\left|\lambda-\lambda^{*}\right| \leq \rho$. Equation (17) has a unique solution $\widehat{e}_{N}$, and as a consequence, $e_{N}$ and $\psi_{N}$ also are unique for $N \geq N_{0}$ and $\left|\lambda-\lambda^{*}\right| \leq \rho$. According to well-known results of interpolation theory and the Stirling formula, it follows that $\left\|r_{N}\right\|_{H}$ goes to zero with spectral accuracy, i.e., $\left\|r_{N}\right\|_{H} \leq \frac{C_{3}}{\sqrt{N}}\left(\frac{C_{2}}{N !}\right)^{N}|x|_{2}$, with $C_{3}:=C_{3}\left(\lambda^{*}, \rho\right), C_{2}:=C_{2}\left(\lambda^{*}, \rho\right)$. Now, from $\left\|e_{N}\right\|_{H} \leq C_{0}\left\|r_{N}\right\|_{H}$ we easily get the bound, where $C_{1}:=C_{0} C_{3}$.

By carrying out the same program as in [19], we obtain the following theorem.

Theorem 2.4. Let $\lambda^{*}$ be an eigenvalue of $A$ with multiplicity $m$. Then, for sufficiently large $N, A_{N}$ has exactly $m$ eigenvalues $\lambda_{N, i}, i=1, \ldots, m$, such that

$$
\max _{i=1, \ldots, m}\left|\lambda^{*}-\lambda_{N, i}\right| \leq\left(\frac{\widehat{C}_{1}}{\sqrt{N}}\right)^{1 / m}\left(\frac{\widehat{C}_{2}}{N}\right)^{N / m},
$$

where $\widehat{C}_{1}, \widehat{C}_{2}$ are constants independent of $N$ and proportional to $\left|\lambda^{*}\right|$.

The convergence of the approximated eigenvalues to the exact ones is faster than $O\left(N^{-k}\right)$ for any $k>0$. This property, also known as spectral accuracy, is the outstanding feature of the pseudospectral approach w.r.t. other techniques that give a fixed order of convergence. For instance, in [41] the convergence rate of the eigenvalues depends on the order of the splines used to discretize $A$ on $E$.

Note that the above results also hold when choosing (14) as the Lobatto points [60].

It is important to underline that there are no "ghost eigenvalues"; i.e., if there is a sequence of approximated eigenvalues that converges, then it converges to an exact eigenvalue of $A$, and the numerical computations confirm that no spurious eigenvalues exist [13, Proposition 3.7]. Finally, we remark that, due to the spectral convergence of the IG approach, the eigenvalues in a ball of radius $\rho$ around the origin can be accurately approximated for sufficiently large $N$ [13, Theorem 5.2]. A procedure for the automatic selection of $N$ is described in [64].

In the pseudospectral approach, it is appropriate to express the polynomial $\varphi_{N}$ in Lagrange form, i.e., $\varphi_{N}(\theta)=\sum_{j=0}^{N} \ell_{j}(\theta) \varphi_{N}\left(\theta_{j}\right), \theta \in[-\tau, 0]$, where $\ell_{j}$ are the Lagrange polynomials relevant to the points (14). Therefore, $A_{N}$ is represented by the matrix $\mathrm{A}_{N} \in \mathbb{R}^{d(N+1) \times d(N+1)}$ with block-entries

$$
\left\{\begin{array}{l}
\left(\mathrm{A}_{N}\right)_{00}=\mathrm{B}+L \ell_{0}, \\
\left(\mathrm{~A}_{N}\right)_{0 j}=L \ell_{j}, \quad i=0, j=0,1, \ldots, N \\
\left(\mathrm{~A}_{N}\right)_{i j}=\ell_{j}^{\prime}\left(\theta_{i}\right) I_{d}, \quad i=1, \ldots, N, j=0,1, \ldots, N
\end{array}\right.
$$

where $\ell_{j}$ are the Lagrange polynomials, $L \ell_{j}: x \in \mathbb{R}^{d} \mapsto L\left(\ell_{j}(\cdot) x\right) \in \mathbb{R}^{d}$, and $\ell_{j}(\cdot) x$ is the function $\left(\ell_{j}(\cdot) x\right)(\theta)=\ell_{j}(\theta) x, \theta \in[-\tau, 0], x \in \mathbb{R}^{d}$.

It is worthwhile to remark that it is possible to apply the pseudospectral method by using the Legendre polynomials as a polynomial basis. In this case, $\varphi_{N}$ is described by its $N+1$ spectral coefficients, and by applying $A$ to $\varphi_{N}$ and imposing the domain condition, we obtain the alternative representation of $A_{N}$ given in [42]. In the paper, the authors propose Legendretau approximations for the solutions of linear (1) and prove the convergence of the method as $N$ goes to infinity. They also derive the discrete characteristic equation without providing proof of eigenvalue convergence. As a result Theorem 2.4 fills the gap. 
3. Polynomial chaos expansions. In this section we set the probabilistic framework and briefly summarize the basic notation of the polynomial chaos (PC) theory. The reader is referred to $[52,59]$ for relevant results and applications.

Let $(\Omega, \Sigma, \mathcal{P})$ be a complete probability space, where $\Omega$ is the event space, $\Sigma$ is the $\sigma$ algebra, and $\mathcal{P}$ is the probability measure. Let $L^{2}(\Omega, \Sigma, \mathcal{P})$ be the Hilbert space of secondorder real-valued random variables defined on the probability space $(\Omega, \Sigma, \mathcal{P})$, equipped with the inner product

$$
\langle Y, Z\rangle_{\Omega}=\int_{\Omega} Y(\omega) Z(\omega) d \mathcal{P}(\omega)
$$

and the induced norm $\|Y\|_{\Omega}=\sqrt{\langle Y, Y\rangle_{\Omega}}$.

Wiener's theory states that a random process with finite second-order moment can be expressed into a convergent series of polynomials in a sequence of Gaussian random variables $\left\{\xi_{i}\right\}_{i=1}^{\infty}$ of the form

$$
Y(\omega)=y_{0} H_{0}+\sum_{\alpha_{1}=1}^{\infty} y_{\alpha_{1}} H_{1}\left(\xi_{\alpha_{1}}(\omega)\right)+\sum_{\alpha_{1}=1}^{\infty} \sum_{\alpha_{2}=1}^{\alpha_{2}} y_{\alpha_{1}, \alpha_{1}} H_{2}\left(\xi_{\alpha_{1}}(\omega), \xi_{\alpha_{2}}(\omega)\right)+\cdots
$$

where $H_{i}$ denotes the set of polynomials of degree $i$ which are mutually orthogonal with regards to the Gaussian measure associated to $\left\{\xi_{i}\right\}_{i=1}^{\infty}[22,63]$. The series is called the $P C$ expansion of $Y$, and its deterministic coefficients, known as chaos coefficients, capture the probabilistic description of the random quantity. In practice, one reduces the representation to a finite number $M$ of random variables, which is governed by the uncertain data, and truncates the PC expansion to order $P$. Specifically all the infinite sums in (19) are replaced by sums over $M$ dimensions with polynomials of degree less than or equal to $p$.

Denote $\xi=\left(\xi_{1}, \ldots, \xi_{M}\right)$ the vector of $M$ independent standard random variables defined on $(\Omega, \Sigma, \mathcal{P})$ with values in the locally compact set $\Xi \subset \mathbb{R}^{M}$ and with joint probabilistic density function $p_{\xi}$, i.e.,

$$
p_{\xi}(\xi)=\prod_{i=1}^{M} p_{i}\left(\xi_{i}\right)
$$

where $p_{i}\left(\xi_{i}\right)$ is the probability density function of $\xi_{i}$. The number $M$ is known as the stochastic dimension. Similarly denote $L^{2}\left(\Xi, \mathcal{B}_{\Xi}, p_{\xi}\right)$ the Hilbert space of second-order real-valued random variables defined on the image probability space $\left(\Xi, \mathcal{B}_{\Xi}, p_{\xi}\right)$, where $\mathcal{B}_{\Xi}$ is the Borel set of $\Xi$. The inner product is given by

$$
\langle Y, Z\rangle_{\Xi}:=\int_{\Xi} Y(\xi) Z(\xi) p_{\xi}(\xi) d \xi
$$

and $\|Y\|_{\Xi}=\sqrt{\langle Y, Y\rangle_{\Xi}}$ is the induced norm. Now consider the $\mathrm{PC}$ basis for $L^{2}\left(\Xi, \mathcal{B}_{\Xi}, p_{\xi}\right)$ given by the set of $M$-dimensional real orthogonal polynomials $\left\{\Psi_{\alpha}\right\}$, where $\alpha=\left(\alpha_{1}, \ldots, \alpha_{M}\right) \in$ $\mathbb{N}_{0}^{M}$ denotes an $M$-dimensional multi-index, $\Psi_{\alpha}$ has total order $|\alpha|=\sum_{i=1}^{M} \alpha_{i}$, and $\Psi_{0}=$ $1,\left\langle\Psi_{\alpha}, \Psi_{\beta}\right\rangle_{\Xi}=\delta_{\alpha \beta}\left\|\Psi_{\alpha}\right\|_{\Xi}^{2}$. 
The $M$-dimensional polynomials $\Psi_{\alpha}$ are constructed via a tensor product of one-dimensional polynomials, i.e.,

$$
\Psi_{\alpha}(\xi)=\Psi_{\alpha_{1}}\left(\xi_{1}\right) \cdots \Psi_{\alpha_{M}}\left(\xi_{M}\right), \alpha \in \mathbb{N}_{0}^{M} .
$$

The original PC expansions consider Hermite polynomials, but the approach has been generalized to other families of orthogonal polynomials, induced by the density function $p_{\xi}$ of the random vector $\xi$ [57]. For example, Legendre polynomials are considered for uniformly distributed random variables, while Laguerre polynomials are used for gamma distribution. Note that the tensored construction of the PC basis (21) allows us to consider also random variables associated with different probability laws.

The random variable $Y \in L^{2}(\Omega, \Sigma, \mathcal{P})$ can be approximated by means of the truncated expansion $Y_{P} \in L^{2}\left(\Xi, \mathcal{B}_{\Xi}, p_{\xi}\right)$,

$$
Y \approx Y_{P}=\sum_{\alpha \in \mathbb{N}_{0}^{M},|\alpha| \leq P} y_{\alpha} \Psi_{\alpha}(\xi)
$$

while with regard to the expectation and variance of $Y$ we obtain, respectively,

$$
\begin{aligned}
E[Y]=\int_{\Omega} Y(\omega) d \mathcal{P}(\omega) \approx E\left[Y_{P}\right] & =\int_{\Omega} Y_{P}(\xi(\omega)) d \mathcal{P}(\omega)=\int_{\Xi} Y_{P}(\xi) p_{\xi}(\xi) d \xi \\
& =\sum_{\alpha \in \mathbb{N}_{0}^{M},|\alpha| \leq P} y_{\alpha} \int_{\Xi} \Psi_{\alpha}(\xi) p_{\xi}(\xi) d \xi=y_{0}
\end{aligned}
$$

and

$$
\begin{aligned}
\operatorname{Var}[Y]=E\left[(Y-E[Y])^{2}\right] \approx \operatorname{Var}\left[Y_{P}\right] & =\int_{\Omega}\left(Y_{P}(\xi(\omega))-E\left[Y_{P}\right]\right)^{2} d \mathcal{P}(\omega) \\
& =\int_{\Xi}\left(Y_{P}(\xi)-E\left[Y_{P}\right]\right)^{2} d p_{\xi}(\xi) d \xi \\
& =\sum_{\alpha \in \mathbb{N}_{0}^{M},|\alpha| \leq P}\left|y_{\alpha}\right|^{2}\left\|\Psi_{\alpha}\right\|_{\Xi}^{2} .
\end{aligned}
$$

In other words we operate in the image probability space $L^{2}\left(\Xi, \mathcal{B}_{\Xi}, p_{\xi}\right)$ instead of the original probability space $L^{2}(\Omega, \Sigma, \mathcal{P})$. We should emphasize that $P$ is the maximal total degree of the polynomial basis, and that the number of terms in $(22)$ is $\frac{(M+P) !}{M ! P !}$, which increases w.r.t. both stochastic dimension $M$ and the expansion degree $P$. The truncated error $Y-Y_{P}$ depends on both $M$ and $P$, and it converges in the mean square sense as $M$ and $P$ go to infinity $[22,52]$. As already pointed out, $M$ is fixed by the parametrization of the model to be studied, while the number $P$ needed to achieve a given error depends on the random variable to represent. The PC representation is computationally efficient when the truncated error rapidly goes to zero as $P$ goes to infinity.

The PC expansion can be used to represent the solution of a mathematical model with uncertain data, that are assumed to be already parametrized by the random vector $\xi$. The probability law of the output solution to be determined is not known a priori, and it is not possible to construct a priori the optimal orthogonal basis. Since the probability law of the data uncertainties to propagate is known, the $\mathrm{PC}$ basis is chosen so as to be optimal w.r.t. 
them. Coupled with suitable numerical methods, the PC expansion of the model solution can be determined and the uncertainty propagation can be quantified.

Finally, we observe that the same representations and approximations can also be extended to complex-valued random variables, random vectors, and processes.

4. Linear uncertain delay differential equations. In this section we define uncertain delay differential equations, and after a suitable abstract reformulation, we study the well-posedness of the associated initial value problem and the mean-square stability of the zero solution. To analyze and quantify the effect induced by the uncertain parameters we propose a numerical technique based on the pseudospectral approach described in section 2 and the SC method.

Let $\mathcal{Y}$ be the Hilbert space of the $\mathbb{R}^{d}$-valued functions on $\Xi$ with all components in $L^{2}\left(\Xi, \mathcal{B}_{\Xi}, p_{\xi}\right)$ equipped with the inner product

$$
\langle Y, Z\rangle_{\mathcal{Y}}:=\int_{\Xi} Y(\xi)^{T} Z(\xi) p_{\xi}(\xi) d \xi
$$

and the induced norm $\|Y\|_{\mathcal{Y}}=\sqrt{\langle Y, Y\rangle_{\mathcal{Y}}}$. Bearing in mind the probabilistic setting in section 3 , we introduce the following definition.

Definition 4.1. A linear uncertain DDE (UDDE) of stochastic dimension $M$ is the parameter dependent $D D E$

$$
\frac{d}{d t} y(t, \xi)=B(\xi) y(t, \xi)+L(\xi) y_{t}(\cdot, \xi), t \geq 0, \text { a.e. } \xi \in \Xi,
$$

where

1. $\xi=\left(\xi_{1}, \ldots, \xi_{M}\right)$ is a random vector of $M$ independent standard random variables defined on $(\Omega, \Sigma, \mathcal{P})$ with values in the locally compact set $\Xi \subset \mathbb{R}^{M}$, and joint probabilistic density function $p_{\xi}$,

2. $B(\xi) \in \mathbb{R}^{d \times d}$ for all realizations $\xi \in \Xi$,

3. $\xi \in \Xi \rightarrow B(\xi) x \in \mathcal{Y}$ for all $x \in \mathbb{R}^{d}$,

4. $\tau>0$ is the maximum delay,

5. for any $t \geq 0$ the history segment $y_{t}(\cdot, \xi)$ belongs to $H$ for all realizations $\xi \in \Xi$, and $y_{t}(\theta, \cdot)$ belongs to $\mathcal{Y}$ for every $\theta \in[-\tau, 0]$,

6. for all realizations $\xi \in \Xi L(\xi): W \rightarrow \mathbb{R}^{d}$ is a linear and bounded delay operator given by

$$
L(\xi) \varphi=\int_{-\tau}^{0} d \eta_{\theta}(\theta, \xi) \varphi(\theta)
$$

where $\eta(\cdot, \xi):[-\tau, 0] \rightarrow \mathbb{R}^{d \times d}$ is a bounded variation matrix,

7. $\xi \in \Xi \rightarrow L(\xi) \varphi \in \mathcal{Y}$ for every $\varphi \in W$.

Here, $\mathcal{Y}$ is the stochastic space, and the $M$-dimensional random vector $\xi$ describes the uncertainty in the parameters of the delay differential equations. In order to treat DDEs with stochastic processes as parameters a preprocessing step, for example, by means of a (truncated) Karhunen-Loève expansion, has to be performed to approximate the stochastic process by a finite set of random variables [52]. 
Given $X \in \mathcal{Y}$ and $\Phi$ such that $\Phi(\cdot, \xi) \in H$ for all realizations $\xi \in \Xi$, and $\Phi(\theta, \cdot) \in \mathcal{Y}$ for all $\theta \in[-\tau, 0]$, the IVP for $(25)$ reads as

$$
\left\{\begin{aligned}
\frac{d}{d t} y(t, \xi) & =\mathrm{B}(\xi) y(t, \xi)+L(\xi) y_{t}(\cdot, \xi), t \geq 0, \text { a.e. } \xi \in \Xi \\
y(0, \xi) & =X(\xi) \\
y_{0} & =\Phi
\end{aligned}\right.
$$

For all realizations $\xi \in \Xi,(27)$ can be viewed as an IVP for a deterministic DDE on the finite-dimensional space $\mathbb{R}^{d}$. Therefore, for a.e. $\xi \in \Xi$ and initial state $(x:=X(\xi), \varphi:=$ $\Phi(\cdot, \xi)) \in E$ we can define the (classical or mild) solution $y(\cdot, \xi ; x, \varphi)$ on $[\tau,+\infty)$. As a function of $t$ for a given $\xi$ the $y(\cdot, \xi)$ is called a sample path or a trajectory of $y$. Moreover we can introduce either the linear and bounded solution operator $T(t, \xi): E \rightarrow E$, which maps the initial state $(x:=X(\xi), \varphi:=\Phi(\cdot, \xi)) \in E$ into the state at time $t \geq 0$, i.e.,

$$
T(t, \xi)(x, \varphi)=\left(y(t, \xi), y_{t}(\cdot, \xi)\right), t \geq 0,
$$

or the associated infinitesimal generator $A(\xi): D(A(\xi)) \subseteq E \rightarrow E$,

$$
\left\{\begin{array}{l}
A(\xi)(x, \varphi)=\left(\mathrm{B}(\xi) x+L(\xi) \varphi, \frac{d}{d \theta} \varphi\right) \\
D(A(\xi))=\left\{(x, \varphi) \in \mathbb{R}^{d} \times W: \varphi(0)=x\right\}
\end{array}\right.
$$

which is closed, densely defined, and unbounded. It is important to observe that the domain of $A(\xi)$ doesn't depend on $\xi$.

To define the solutions and investigate the well-posedness of the IVP (27), first we introduce further assumptions on the families of matrices $\mathrm{B}(\xi)$ and operators $L(\xi)$. Then we reformulate the linear UDDE (25) as a linear abstract DDE (ADDE) on $\mathcal{Y}$. In fact, similar to the finite-dimensional case, the basic definitions and results on the solvability of the Cauchy problem recalled in section 2 also apply to infinite dimensions [6]. Moreover the norm-stability of the zero solution of the linear UDDE (25), also called mean-square stability [48], can be investigated by the spectral theory of semigroup (see section 4.2). Finally we remark that the principle of linearized stability can be also applied [6]. General ADDEs on $C([-\tau, 0], \mathcal{Y})$ are addressed in $[2,30]$.

4.1. Reformulation as abstract delay differential equation. Let $\mathcal{H}:=L^{2}(-\tau, 0 ; \mathcal{Y})$ be the history space for the UDDE (25) equipped with the inner product

$$
\langle\Phi, \Psi\rangle_{\mathcal{H}}=\int_{-\tau}^{0}\langle\Phi(\theta, \cdot), \Psi(\theta, \cdot)\rangle_{\mathcal{Y}} d \theta, \Phi, \Psi \in \mathcal{H}
$$

and the induced norm

$$
\|\Phi\|_{\mathcal{H}}=\sqrt{\left(\int_{-\tau}^{0}\|\Phi(\theta, \cdot)\|_{\mathcal{Y}}^{2} d \theta\right)}=\sqrt{\left(\int_{-\tau}^{0} \int_{\Xi}|\Phi(\theta, \xi)|_{2}^{2} p_{\xi}(\xi) d \xi d \theta\right)} .
$$

Here we adopt the notation $\Phi(\theta, \xi)$ for all the functions $\Phi \in \mathcal{H}$. According to Fubini's theorem

$$
\|\Phi\|_{\mathcal{H}}=\left(\int_{\Xi} \int_{-\tau}^{0}|\Phi(\theta, \xi)|_{2}^{2} d \theta p_{\xi}(\xi) d \xi\right)^{\frac{1}{2}}=\left(\int_{\Xi}\|\Phi(\cdot, \xi)\|_{H}^{2} p_{\xi}(\xi) d \xi\right)^{\frac{1}{2}},
$$


and $\mathcal{H} \sim L^{2}(\Xi, H)[6]$. Moreover, we define the space $\mathcal{W}:=W^{1,2}(-\tau, 0 ; \mathcal{Y})$. Hereafter we also assume that

$$
\begin{gathered}
\|\mathrm{B}(\xi)\| \leq \beta, \text { a.e. } \xi \in \Xi, \\
\|L(\xi)\| \leq \ell, \text { a.e. } \xi \in \Xi .
\end{gathered}
$$

Proposition 4.2. Assume that the family of matrices $\{B(\xi)\}_{\xi \in \Xi}$ satisfies (30). Then the linear operator $\mathcal{B}: D(\mathcal{B}) \subseteq \mathcal{Y} \rightarrow \mathcal{Y}$ induced on $\mathcal{Y}$ by the family $\{B(\xi)\}_{\xi \in \Xi}$ given by

$$
\left\{\begin{array}{l}
(\mathcal{B} Y)(\xi):=B(\xi) Y(\xi), \text { a.e. } \xi \in \Xi \\
D(\mathcal{B})=\{Y \in \mathcal{Y}: \xi \in \Xi \rightarrow B(\xi) Y(\xi) \in \mathcal{Y}\}
\end{array}\right.
$$

is bounded. Moreover, $D(\mathcal{B})=\mathcal{Y}$.

Proof. For all functions $Y \in D(\mathcal{B})$ we can see that

$$
\begin{aligned}
\|\mathcal{B} Y\|_{\mathcal{Y}}^{2} & =\int_{\Xi}|\mathrm{B}(\xi) Y(\xi)|_{2}^{2} p_{\xi}(\xi) d \xi \\
& \leq \int_{\Xi}\|\mathrm{B}(\xi)\|^{2}|Y(\xi)|_{2}^{2} p_{\xi}(\xi) d \xi \\
& \leq \beta^{2}\|Y\|_{\mathcal{Y}}^{2},
\end{aligned}
$$

and the thesis follows.

Proposition 4.3. If the linear operator $\mathcal{B}: \mathcal{Y} \rightarrow \mathcal{Y}$ induced on $\mathcal{Y}$ by the family of matrices $\{B(\xi)\}_{\xi \in \Xi}$ given in (32) is bounded, then (30) holds.

Proof. Since $\mathcal{B}$ is bounded, there is a constant $C \geq 0$ such that $\|\mathcal{B} Y\|_{\mathcal{Y}}^{2} \leq C\|Y\|_{\mathcal{Y}}^{2}$ for all $Y \in \mathcal{Y}$. Consider the set $\Xi(\beta)=\{\xi \in \Xi \mid\|\mathrm{B}(\xi)\|>\beta\}$ for some $\beta>0$. For any $\xi \in \Xi(\beta)$ we find $v(\xi) \in \mathbb{R}^{d},|v(\xi)|_{2}=1$ such that $\beta \leq|\mathrm{B}(\xi) v(\xi)|_{2}$. Let $Y(\xi):=\chi_{\Xi(\beta)} v(\xi)$, where $\chi_{\Xi(\beta)}$ is the characteristic function of the set $\Xi(\beta)$. We have

$$
\beta^{2} \int_{\Xi(\beta)} p_{\xi}(\xi) d \xi \leq \int_{\Xi}|\mathrm{B}(\xi) Y(\xi)|_{2}^{2} p_{\xi}(\xi) d \xi \leq C \int_{\Xi(\beta)} p_{\xi}(\xi) d \xi
$$

If we select $\beta^{2}>C$, we obtain $\int_{\Xi(\beta)} p_{\xi}(\xi) d \xi=0$, which implies that $\|\mathrm{B}(\xi)\| \leq \beta$ a.e.

We remark that the linear bounded operator $\mathcal{B}$ in (32) is the infinitesimal generator of the semigroup $\{\mathcal{S}(t)\}_{t \geq 0}$, where $\mathcal{S}(t): \mathcal{Y} \rightarrow \mathcal{Y}$ is the multiplication operator given by

$$
(\mathcal{S}(t) Y)(\xi)=e^{t \mathrm{~B}(\xi)} Y(\xi), \quad \xi \in \Xi, Y \in \mathcal{Y}, t \geq 0 .
$$

The semigroup $\{\mathcal{S}(t)\}_{t \geq 0}$ is uniformly continuous; i.e., the map $t \mapsto \mathcal{S}(t)$ is continuous in the uniform operator topology [24].

Similarly, we are able to prove the following propositions. 
Proposition 4.4. Assume that the family $\{L(\xi)\}_{\xi \in \Xi}$ of linear and bounded operator $L(\xi)$ : $W \rightarrow \mathbb{R}^{d}$ satisfies (31). Then the linear operator $\mathcal{L}: D(\mathcal{L}) \subseteq \mathcal{W} \rightarrow \mathcal{Y}$ induced on $\mathcal{W}$ by the

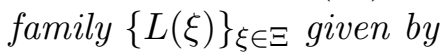

$$
\left\{\begin{array}{l}
\mathcal{L} \Phi(\xi):=L(\xi) \Phi(\cdot, \xi), \text { a.e. } \xi \in \Xi \\
D(\mathcal{L})=\{\Phi \in \mathcal{W}: \quad \xi \in \Xi \rightarrow L(\xi) \Phi(\cdot, \xi) \in \mathcal{Y}\}
\end{array}\right.
$$

is bounded. Moreover $D(\mathcal{L})=\mathcal{W}$.

Proposition 4.5. If the linear operator $\mathcal{L}: \mathcal{W} \rightarrow \mathcal{Y}$ induced on $\mathcal{W}$ by the family of operators $\{L(\xi)\}_{\xi \in \Xi}$ given in (26) is bounded, then (31) holds.

Both the operators $\mathcal{B}$ and $\mathcal{L}$ are multiplication operators. By defining

$$
Y(t+\theta)(\xi):=y(t+\theta, \xi), \quad t \geq 0, \theta \in[-\tau, 0], \text { a.e. } \xi \in \Xi,
$$

the UDDE (25) can be recast as the following linear ADDE on $\mathcal{Y}$ :

$$
Y^{\prime}(t)=\mathcal{B} Y(t)+\mathcal{L} Y_{t}, \quad t \geq 0,
$$

where both the operators $\mathcal{B}$ and $\mathcal{L}$ are bounded.

Now all the steps in section 2 for DDE (1) can also be followed in the case of ADDE (34): classical and mild solutions of the associated initial value problem can be defined, and the well-posedness of the problem can be proved. Moreover, the semigroup of solution operators and the infinitesimal generator can be introduced [6].

For any $X \in \mathcal{Y}$ and $\Phi \in \mathcal{H}$, the IVP for the linear ADDE (34) reads as

$$
\left\{\begin{aligned}
Y^{\prime}(t) & =\mathcal{B} Y(t)+\mathcal{L} Y_{t}, \quad t \geq 0 \\
Y(0) & =X \\
Y_{0} & =\Phi
\end{aligned}\right.
$$

To study the existence and uniqueness of solutions of the IVP (35), we have to define what a solution is. We carry on as in section 2 .

Definition 4.6. We say that $Y$ is a classical solution of the IVP (35) on $[\tau,+\infty)$ if $Y \in$ $C([-\tau,+\infty), \mathcal{Y}) \cap C^{1}([0,+\infty), \mathcal{Y}), Y_{t} \in \mathcal{W}$ for all $t \geq 0$, and $Y$ satisfies (35) for all $t \geq 0$.

Definition 4.7. We say that $Y$ is a mild solution of the IVP (35) on $[\tau,+\infty)$ if $\int_{0}^{t} Y_{s} d s \in \mathcal{W}$ for all $t \geq 0, Y$ satisfies the integral abstract formulation

$$
Y(t)=X+\mathcal{B} \int_{0}^{t} Y(s) d s+\mathcal{L} \int_{0}^{t} Y_{s} d s, \quad t \geq 0,
$$

and the initial condition $Y_{0}=\Phi$.

According to [6, Theorem 3.23] the IVP (35) has a unique (classical or mild) solution. The extended state is

$$
U(t):=\left(Y(t), Y_{t}\right), \quad t \geq 0
$$


and it belongs to the state space $\mathcal{E}:=\mathcal{Y} \times \mathcal{H}$, equipped with the norm

$$
\|(X, \Phi)\|_{\mathcal{E}}=\sqrt{\|X\|_{\mathcal{Y}}^{2}+\|\Phi\|_{\mathcal{H}}^{2}}
$$

It is important to observe that according to Fubini's theorem $\mathcal{E} \sim L^{2}\left(\Xi, \mathbb{R}^{d} \times H\right)$.

The solution operator $\mathcal{T}(t): \mathcal{E} \rightarrow \mathcal{E}$ is the linear and bounded operator that associates the state $\left(Y(t), Y_{t}\right)$ at time $t$ to the initial state $(X, \Phi)$, i.e.,

$$
\mathcal{T}(t)(X, \Phi)=\left(Y(t), Y_{t}\right), \quad t \geq 0 .
$$

The family $\{\mathcal{T}(t)\}_{t \geq 0}$ forms a $C_{0}$-semigroup. The associated infinitesimal generator $\mathcal{A}$ : $D(\mathcal{A}) \subseteq \mathcal{E} \rightarrow \mathcal{E}$ is the linear unbounded operator given by

$$
\left\{\begin{array}{l}
\mathcal{A}(X, \Phi)=\left(\mathcal{B} X+\mathcal{L} \Phi, \frac{d}{d \theta} \Phi\right) \\
D(\mathcal{A})=\{(X, \Phi) \in \mathcal{Y} \times \mathcal{W}: \Phi(0)=X\}
\end{array}\right.
$$

Given $(X, \Phi) \in \mathcal{E}$, the $\mathrm{ACP}$ associated with $\mathcal{A}$ on the space $\mathcal{E}$ reads as

$$
\left\{\begin{array}{l}
U^{\prime}(t)=\mathcal{A} U(t), \quad t \geq 0 \\
U(0)=(X, \Phi)
\end{array}\right.
$$

Again, classical and mild solutions of (39) can be defined, and it can be proved that (35) and (39) are equivalent [6].

4.2. The semigroup approach. The abstract reformulation (34) allows us to address also the norm-stability of the zero solution of (25) through the semigroup theory [24, 31]. With regard to the spectral analysis, it is necessary to work on $\mathbb{C}$, and we implicitly assume that $\mathcal{H}, \mathcal{Y}$, and all the operators involved have been complexified. Since we are in the infinitedimensional case, the eventual compactness of the semigroup will generally be lacking, and the spectral bound and the growth bound may be different. In this more general context the asymptotic behavior of the semigroup cannot be described by analyzing the spectrum of the infinitesimal generator, and the notions of essential and nonessential are important. But in the particular case of UDDE the spectral bound and the growth bound coincide, and consequently the asymptotic behavior of the solutions can be described by the location of the infinitesimal generator spectrum in the complex plane. The key is that all the operators can be represented by a family of operators indexed by $\xi \in \Xi$. In fact, the introduction of the family of operators (28) and (29) allows us to express the solution operator (37) as

$$
(\mathcal{T}(t) \Phi)(\cdot, \xi)=T(t, \xi) \Phi(\cdot, \xi), \text { a.e. } \xi \in \Xi,
$$

whereas the associated infinitesimal generator (38) permits the representation

$$
(\mathcal{A} \Phi)(\cdot, \xi)=A(\xi) \Phi(\cdot, \xi), \text { a.e. } \xi \in \Xi,
$$

since for all realizations $\xi \in \Xi$ the function $\Phi(\cdot, \xi)$ belongs to $D(A(\xi))$. The operators $\mathcal{T}(t)$ and $\mathcal{A}$ are multiplication operators, and since $\mathcal{T}(t)$ is a multiplication operator for all $t \geq 0$, we call $\{\mathcal{T}(t)\}_{t \geq 0}$ an SO-multiplication semigroup. 
Since $\{\mathcal{S}(t)\}_{t \geq 0}$ in (33) is uniformly continuous, and from (26) the operator $\mathcal{L}$ is given by the Riemann-Stieltjes integral of a bounded variation function, we can apply $[6$, Theorem 4.11] in order to obtain $s(\mathcal{A})=\omega_{0}(\mathcal{T})$. If $s(\mathcal{A})<0$, then the SO-multiplication semigroup is exponentially asymptotically stable and every classical or mild solution of the linear UDDEs is mean-square stable. The next theorem relates the spectrum of (38) to the spectrum of the family of operators (29). Similar results were derived in [29] under continuity assumptions on the family of operators and in [28] in the case of partial differential operators arising in hydrodynamics.

Theorem 4.8. Let $\mathcal{A}$ be the infinitesimal generator (38) of the SO-multiplication semigroup $\{\mathcal{T}(t)\}_{t \geq 0}$. Then

(i) $\sigma(\mathcal{A})=\overline{\bigcup_{\text {a.e. } \xi \in \Xi} \sigma(A(\xi))}$,

(ii) $s(\mathcal{A})=$ ess-sup $_{\xi \in \Xi} \Re(\sigma(A(\xi))$,

where the operators $A(\xi)$ are defined in (29).

Proof. (i) As a first step we will describe the resolvent set $\rho(\mathcal{A})$ of $\mathcal{A}$ in terms of the resolvent sets of the family of the operators $\mathcal{A}(\xi)$. In particular we will prove that

$$
\rho(\mathcal{A})=\bigcap_{\text {a.e. } \xi \in \Xi} \rho(A(\xi)) .
$$

By standard analysis, we obtain that a necessary and sufficient condition for $\lambda \in \rho(\mathcal{A})$ is that the operator $\Delta(\lambda): \mathcal{Y} \rightarrow \mathcal{Y}$ given by

$$
\Delta(\lambda):=\lambda-\mathcal{B}-\mathcal{L} e^{\lambda}
$$

is invertible [6, p. 56]. Moreover, it is easy to see that $\Delta(\lambda)$ is a multiplication operator induced by the family of matrices $\Delta(\lambda, \xi)=I-B(\xi)-L(\xi) e^{\lambda}, \xi \in \Xi$.

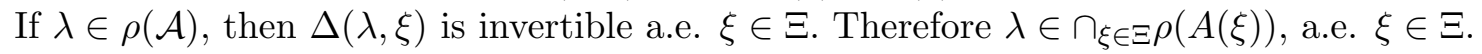

If $\lambda \in \rho(A(\xi))$ a.e. $\xi \in \Xi$, then the matrix $\Delta(\lambda, \xi)$ is invertible for a.e. $\xi \in \Xi$. Hence there exists $\epsilon>0$ such that the set $B=\left\{\xi \in \Xi: \sigma(\Delta(\lambda, \xi)) \cap \mathbb{D}_{\epsilon}\right\}$ has measure zero, where $\mathbb{D}_{\epsilon}$ is the open disk in $\mathbb{C}$ with center 0 and radius $\epsilon$. Let $\nu:=\inf \{|\operatorname{det}(\Delta(\lambda, \boldsymbol{\xi}))|: \xi \in \Xi \backslash B\}$. Then $\left\|\Delta(\lambda, \xi)^{-1}\right\| \leq \frac{\|\Delta(\lambda, \xi)\|^{d-1}}{|\operatorname{det}(\Delta(\lambda, \xi))|} \leq \frac{1}{\nu}\|\Delta(\lambda)\|$, and the family $\Delta(\lambda, \xi)^{-1}$ is essentially bounded. Therefore we can define $\Delta(\lambda)^{-1}$ for $\lambda \in \rho(\mathcal{A})$.

Since the spectrum is the complementary set of $\rho(\mathcal{A})$, and it is closed, the first point of the theorem is completed.

(ii) This is a consequence of (i).

4.3. The IG approach for uncertain delay differential equations. In order to analyze and quantify the effect induced by the uncertain parameters on the norm stability of the zerosolution of linear UDDEs $(25)$, which is described by $s(\mathcal{A})$, we combine the IG approach for linear deterministic DDE (25) with the SC method applied to the resulting random eigenvalue problem. The SC method extends the definition of collocation methods to stochastic problems. Selecting a set of collocations points in the random space, the SC method solves a deterministic problem at each point and then recovers the solution statistics. The facility of implementation is one of its advantages. Basic ideas and results behind the SC method can be found in [52, 59]. 
Given $\lambda \in \sigma(\mathcal{A})$ and $\epsilon>0$, we know from Theorem 4.8 that there exists $\lambda^{*} \in \sigma\left(A\left(\xi^{*}\right)\right)$ for $\xi^{*} \in \Xi$ such that $\left|\lambda-\lambda^{*}\right| \leq \epsilon$. Let us assume that $\lambda^{*}$ has multiplicity $m$, and let $A_{N}\left(\xi^{*}\right)$ be the finite-dimensional operator obtained by applying the IG approach to $A\left(\xi^{*}\right)$. Then Theorem 2.4 ensures that, for a sufficiently large $N, A_{N}\left(\xi^{*}\right)$ has exactly $m$ eigenvalues $\lambda_{N}^{i}, i=1, \ldots, m$, such that

$$
\max _{i=1, \ldots, m}\left|\lambda-\lambda_{N}^{i}\right| \leq \epsilon+\left(\frac{\widehat{C}_{1}}{\sqrt{N}}\right)^{1 / m}\left(\frac{\widehat{C}_{2}}{N}\right)^{N / m} \leq 2 \epsilon
$$

where $\widehat{C}_{1}, \widehat{C}_{2}$ are constants independent of $N$ and proportional to $\left|\lambda^{*}\right| \leq|\lambda|+\epsilon$.

As already pointed out in section 2 , the spectral convergence and the boundedness of the linear operator $\mathcal{L}$ allow us to determine a small $N$, such that the eigenvalues of $A(\xi)$ in a ball of radius $\rho$ around the origin are approximated by the eigenvalues of $A_{N}(\xi)$ with the prescribed accuracy a.e. $\xi \in \Xi$.

Now, by solving the random eigenvalue problem

$$
\left\{\begin{array}{l}
A_{N}(\xi) V_{N}(\xi)=\Lambda_{N}(\xi) V_{N}(\xi) \\
V_{N}(\xi)^{H} V_{N}(\xi)=1
\end{array}\right.
$$

for the random matrix $A_{N}(\xi)$ obtained by applying the IG approach to the operator (29) for a fixed $N$, we can construct the random variable

$$
S_{N}(\xi)=\max _{\Lambda_{N}(\xi) \in \sigma\left(A_{N}(\xi)\right)} \mathcal{R} e\left(\Lambda_{N}(\xi)\right) \text {, a.e. } \xi \in \Xi
$$

and, from point (ii) of Theorem 4.8, the following approximation:

$$
s(\mathcal{A}) \approx \operatorname{ess}_{-\sup _{\xi \in \Xi}} S_{N}(\xi) .
$$

Proposition 4.9. We have that $\Lambda_{N}(\xi)$ and $S_{N}(\xi)$ are second-order functions on $\Xi$, respectively, with complex and real values.

Proof. Denote as $v^{H}$ the complex conjugate of $v$. Since $V_{N}(\xi)^{H} V_{N}(\xi)=1$ a.e. $\xi \in \Xi$, we can conclude that all the components $V_{N, i}(\xi)$ of $V_{N}(\xi)$ are second-order complex-valued functions on $\Xi$. The definition of the matrix $A_{N}(\boldsymbol{\xi})$ and the assumptions (30), (31) ensure that all the elements $A_{N}(\xi) V_{N}(\xi)$ are second-order complex-valued functions on $\Xi$. Hence by

$$
\left|\Lambda_{N}(\xi)\right|^{2}=\left(A_{N}(\xi) V_{N}(\xi)\right)^{H}\left(A_{N}(\xi) V_{N}(\xi)\right)
$$

and the Cauchy-Schwarz inequality, it follows that $\Lambda_{N}(\xi)$ is a complex-valued second-order function on $\Xi$. Finally, since the maximum of second-order real-valued functions is again a second-order real-valued function, we obtain the desired statement.

Finally, we apply the SC method to (43) according to the following steps.

1. For a given integer $P$, we construct the set $\Xi_{P}$ of $n:=(P+1)^{M}$ distinct collocation points $\xi_{\beta} \in \Xi_{P}$ using tensored grids, i.e., $\xi_{\beta}=\left(\xi_{\beta_{1}}, \ldots, \xi_{\beta_{M}}\right)$ with $\beta=\left(\beta_{1}, \ldots, \beta_{M}\right) \in$ $\mathbb{N}_{0}^{M}$ such that $0 \leq \beta_{i} \leq P, i=1, \ldots, M$. 
2. For each $\xi_{\beta} \in \Xi_{P}$, we solve the deterministic eigenvalue problem

$$
A_{N}\left(\xi_{\beta}\right) v_{N, \beta}=\lambda_{N, \beta} v_{N, \beta}
$$

to compute

$$
S_{\beta}=\max _{\lambda_{N, \beta} \in \sigma\left(A_{N}\left(\xi_{\beta}\right)\right)} \mathcal{R} e\left(\lambda_{N, \beta}\right)
$$

3. We estimate

$$
s(\mathcal{A}) \approx \max _{\substack{\beta \in \mathbb{N}^{M}, 0 \leq \beta_{i} \leq P \\ i=1, \ldots, M}} S_{\beta} .
$$

4. We recover the chaos coefficients $s_{\alpha}$ of the algebraic polynomial of degree $P$ in each of the components $\xi_{i}, i=1, \ldots, M$,

$$
S_{N, P}(\xi)=\sum_{\substack{\alpha \in \mathbb{N}^{M}, 0 \leq \alpha_{i} \leq P, i=1, \ldots, M}} s_{\alpha} \Psi_{\alpha}(\xi),
$$

via tensored $(P+1)$-point Gaussian quadrature, to approximate the statistical moments, e.g., expectation and variance, of $S_{N}$.

Here the subscript $P$ in $S_{N, P}$ stresses the maximum degree of each of the components of the polynomial, whereas its total degree is $M \times P$. In contrast to the one-dimensional case, multidimensional interpolation is not usually treated in the numerical texts; a basic introduction can be found in [40], whereas results on multivariate interpolation error and Jackson's are given in [23] and [55], respectively. The properties of the underlying onedimensional interpolation can be maintained by using the tensor product construction. The convergence rate of $S_{N, P}$ to $S_{N}$ w.r.t. $P$ depends on the stochastic dimension $M$ and the regularity of $S_{N}$. For large dimension $M$ the convergence can be very slow w.r.t. the total number $n$ of collocation points, and moreover, the SG method based on tensored grids becomes computationally expensive. This is the well-known curse of dimensionality. For this reason, tensor product construction is mostly used for low to moderate stochastic dimension $M$. The reduction of complexity can be achieved by using sparse grids, and further comments and results can be found in the books $[52,59]$ and the references therein. SG has been proposed to solve random eigenvalue problems for real symmetric random matrices in [36]. It is worthwhile to remark that the SG method also works for small $M$. Typically Monte Carlo sampling methods are an appropriate choice when $M \geq 10$. The computational cost also depends on the dimension of the matrix $A_{N}$. It is important to emphasize that the total computational cost could be significantly reduced by solving the deterministic eigenvalue problems (44) in parallel.

5. Numerical results. In this section we test our numerical approach on a number of "academic" examples of UDDEs of a small stochastic dimension $M$. The uncertain parameters are described by uniformly distributed random variables, for which we assume to already know the polynomial approximation as in [36]. Therefore, the basis for $L^{2}(\Xi, \mathbb{R})$ is given by the tensor product of Legendre polynomials. For a given $P$ the collocation points are constructed by using $P+1$ tensored Legendre points. Then the total number of collocation 


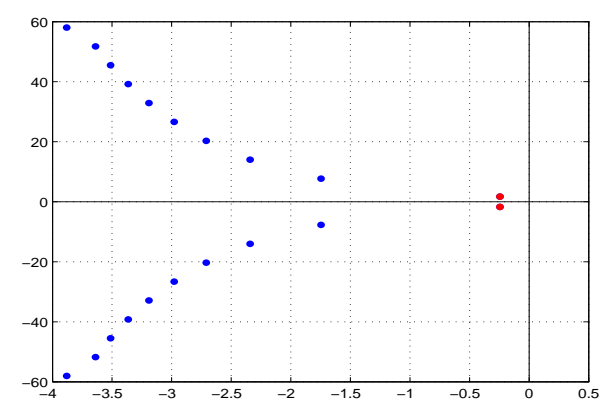

Figure 1. Rightmost eigenvalues of (46) using $a=-0.5, b=-0.5 e$, and $N=20$.

points is $n=(P+1)^{M}$, whereas the maximum degree of $S_{N, P}$ is $M \times P$. It is known that in Monte Carlo methods the statistics based on a sample set of dimension $n$ converge to the true values as $1 / \sqrt{n}$ for sufficiently large $n$, according to the law of large numbers. This low convergence rate is the main limitation in using the Monte Carlo method. To assess the efficiency of our approach, we experimentally investigate the convergence w.r.t. $P$ of the mean and the variance of the stability indicator $S_{N}$. To construct the matrix and solve (44) at the collocation points, we adapt the MATLAB codes available in [19].

Consider the scalar DDE

$$
\frac{d}{d t} y(t)=a y(t)+b y(t-1), \quad t \geq 0,
$$

with $a=-0.5, b=-0.5 e$. By applying the IG approach of section 2 with $N=20$, the computed rightmost pair of eigenvalues is $-0.245203898536 \pm 1.71803022843 i$ (see Figure 1), and the zero solution is asymptotically stable. We emphasize that in [36] the authors consider random symmetric matrices, which have only real eigenvalues. Hereafter, if not differently specified, we assume $N=20$. To quantify the effect on the stability of the uncertain data

$$
\begin{aligned}
& \xi=\left(\xi_{1}, \xi_{2}\right), \quad \xi_{1}, \xi_{2} \sim \mathcal{U}[-1,1], \\
& a(\xi)=-0.5+\frac{\xi_{1}}{2}, \quad b(\xi)=-0.5 e+\frac{\xi_{2}}{2},
\end{aligned}
$$

we consider the following linear UDDE of stochastic dimension $M=2$ :

$$
\frac{d}{d t} y(t, \xi)=a(\xi) y(t, \xi)+b(\xi) y(t-1, \xi), \quad t \geq 0 .
$$

We first apply the classical Monte Carlo sampling method to $S_{N}$. We generate $n=(P+1)^{M}$ random realizations $S_{i}, i=1, \ldots, n$, of $S_{N}$, and we estimate the first two statistical moments using $E_{n}=\frac{\sum_{i=1}^{N} S_{i}}{n}$ and $V_{n}=E_{n}\left(S_{N}-E_{n}\right)^{2}$. The results are given in Table 1. Note that the dimension $n$ of the sample set is equal to the number of the collocation points in section 4.3.

Now we consider the numerical method of section 4.3 , and we compute the mean $E_{N, P}:=$ $E\left[S_{N, P}\right]$ and the variance $V_{N, P}:=\operatorname{Var}\left[S_{N, P}\right]$ of the numerical stability indicator $S_{N, P}$ for the UDDE (46). Varying $P$, we get the numerical results in Table 2, where the errors are computed w.r.t. the values $E_{N, 40}=-0.2632158062801188, V_{N, 40}=3.160813907441763 e-02$. 
Table 1

Numerical results of the Monte Carlo method for the UDDE (46) using data (45) and $N=20$.

\begin{tabular}{|c|c|c|}
\hline$n$ & $E_{n}$ & $V_{n}$ \\
\hline 25 & -0.360817 & 0.029511 \\
100 & -0.265429 & 0.021630 \\
225 & -0.276423 & 0.028096 \\
400 & -0.210894 & 0.028528 \\
10000 & -0.266186 & 0.029296 \\
\hline
\end{tabular}

Table 2

Numerical results for the UDDE (46) using data (45) and $N=20$.

\begin{tabular}{|c|c|c|c|c|}
\hline$P$ & $E_{N, P}$ & $\left|E_{N, P}-E_{N, 40}\right|$ & $V_{N, P}$ & $\left|V_{N, P}-V_{N, 40}\right|$ \\
\hline 5 & -0.2632157770148609 & $2.93 \mathrm{e}-08$ & 0.031608092975692 & $4.61 \mathrm{e}-08$ \\
10 & -0.2632158062794730 & $6.46 \mathrm{e}-13$ & 0.031608139074244 & $1.74 \mathrm{e}-13$ \\
15 & -0.2632158062801199 & $1.11 \mathrm{e}-15$ & 0.031608139074415 & $2.47 \mathrm{e}-15$ \\
20 & -0.2632158062801233 & $4.55 \mathrm{e}-15$ & 0.031608139074419 & $1.11 \mathrm{e}-15$ \\
\hline
\end{tabular}

In Figure 2 the error plot shows the spectral convergence behavior [21]. Moreover we get the estimate $\operatorname{Prob}\left\{S_{N, P}<0\right\}=0.936$ when $P=40$.

The results in Tables 1 and 2 confirm expectations: the Monte Carlo method is robust but its convergence rate is slow, whereas the SC method takes advantage of the smoothness of $S_{N}$ and provides very accurate results. To experimentally investigate how the regularity of $S_{N}$ affects the convergence rates of mean and variance, we consider the UDDE (46) and uncertain data

$$
\begin{aligned}
& \xi=\left(\xi_{1}, \xi_{2}\right), \quad \xi_{1}, \xi_{2} \sim \mathcal{U}[-1,1], \\
& a(\xi)=-0.5+\frac{\xi_{1}+1}{2}, \quad b(\xi)=-0.5 e+\xi_{2} .
\end{aligned}
$$

Varying $P$, the numerical results given in Table 3, where the errors are computed w.r.t. the values $E_{N, 80}=-0.1637698319566630, V_{N, 80}=0.1162072745963336$, show a lower accuracy w.r.t. to the previous case. Note that $\operatorname{Prob}\left\{S_{N, P}<0\right\}=0.603$ when $P=80$.

Table 3

Numerical results for the UDDE (46) using data (47) and $N=20$.

\begin{tabular}{|c|c|c|c|c|}
\hline$P$ & $E_{N, P}$ & $\left|E_{N, P}-E_{N, 80}\right|$ & $V_{N, P}$ & $\left|V_{N, P}-V_{N, 80}\right|$ \\
\hline 5 & -0.158494 & $5.27 \mathrm{e}-03$ & 0.114104 & $2.10 \mathrm{e}-03$ \\
10 & -0.162381 & $1.39 \mathrm{e}-03$ & 0.115432 & $7.75 \mathrm{e}-04$ \\
20 & -0.163878 & $1.23 \mathrm{e}-04$ & 0.116288 & $8.05 \mathrm{e}-05$ \\
30 & -0.163761 & $8.49 \mathrm{e}-06$ & 0.116205 & $2.16 \mathrm{e}-06$ \\
\hline
\end{tabular}

Now let us consider the UDDE of stochastic dimension $M=3$ :

$$
\frac{d}{d t} y(t, \xi)=a(\xi) y(t, \xi)+b\left(\xi_{2}\right) y(t-\tau(\xi), \xi), \quad t \geq 0,
$$

where

$$
\begin{aligned}
& \xi=\left(\xi_{1}, \xi_{2}, \xi_{3}\right), \quad \xi_{1}, \xi_{2}, \xi_{3} \sim \mathcal{U}[-1,1], \\
& a(\xi)=-0.5+\frac{\xi_{1}}{2}, \quad b(\xi)=-0.5 e+2 \xi_{2}, \quad \tau(\xi)=1+0.5 \xi_{3}+0.5 \xi_{3}^{2} .
\end{aligned}
$$



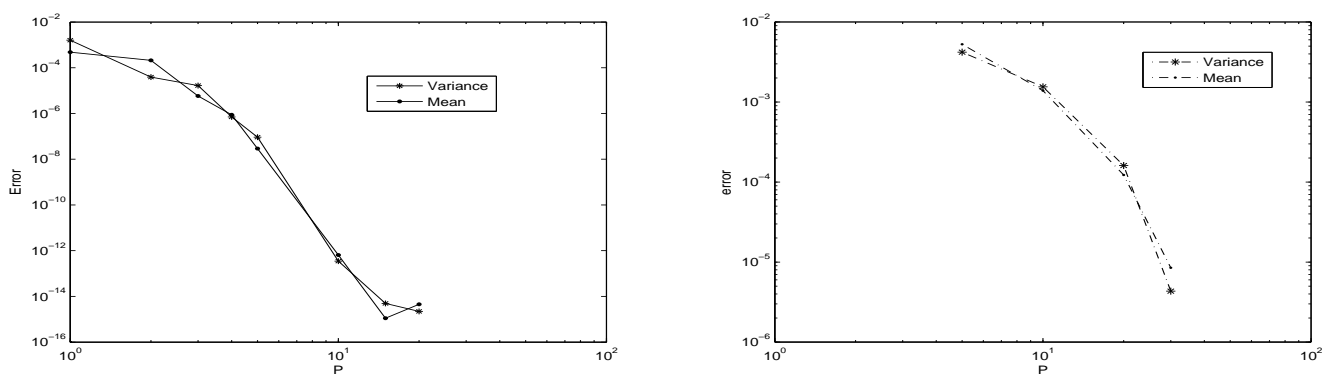

Figure 2. Errors for the UDDE (46) using data (45) (left), data (47) (right), and $N=20$.

The numerical results in Table 4 and the loglog plots in Figure 3 show the spectral convergence of the errors computed w.r.t. $E_{20,20}=-0.2632012891827310, V_{20,20}=3.164449449949752 e-$ 02 .

Table 4

Numerical results for the UDDE (48) using data (49) and $N=20$.

\begin{tabular}{|c|c|c|c|c|}
\hline$P$ & $E_{N, P}$ & $\left|E_{N, P}-E_{N, 20}\right|$ & $V_{N, P}$ & $\left|V_{N, P}-V_{N, 20}\right|$ \\
\hline 5 & -0.2632012599175010 & $2.93 \mathrm{e}-08$ & 0.031644448400687 & $4.61 \mathrm{e}-08$ \\
10 & -0.2632012891819914 & $7.39 \mathrm{e}-13$ & 0.031644494499311 & $1.86 \mathrm{e}-13$ \\
15 & -0.2632012891827369 & $5.88 \mathrm{e}-15$ & 0.031644494499502 & $4.47 \mathrm{e}-15$ \\
\hline
\end{tabular}

Finally, let us consider the UDDE of stochastic dimension $M=3$ and both discrete and distributed delays:

$$
\frac{d}{d t} y(t, \xi)=a(\xi) y(t, \xi)+b(\xi) y(t-1, \xi)+\int_{-1}^{0} e^{5 \theta} c(\xi) \cos (4 \pi \theta) y_{t}(\theta, \xi) d \theta,
$$

where

$$
\begin{array}{ll}
\xi=\left(\xi_{1}, \xi_{2}, \xi_{3}\right), & \xi_{i} \sim \mathcal{U}[-1,1], \quad i=1,2,3, \\
a(\xi)=-0.5+\frac{\xi_{1}}{2}, & b(\xi)=-0.5 e+0.5 \xi_{2}, \quad c(\xi)=\xi_{3} .
\end{array}
$$

Again, the results in Table 5 and the loglog plots in Figure 3 suggest a spectral convergence of the errors computed w.r.t. $E_{N, 20}=-2.632012891829257 e-01, V_{N, 20}=3.164449449950082 e-$ 02. Moreover $\operatorname{Prob}\left\{S_{N, P}<0\right\}=0.936$ when $P=20$.

Table 5

Numerical results for the UDDE (50) using data (51) and $N=20$.

\begin{tabular}{|c|c|c|c|c|}
\hline$P$ & $E_{N, P}$ & $\left|E_{N, P}-E_{N, 20}\right|$ & $V_{N, P}$ & $\left|V_{N, P}-V_{N, 20}\right|$ \\
\hline 5 & -0.2632012599175010 & $2.93 \mathrm{e}-08$ & $3.164444840068725 \mathrm{e}-02$ & $4.61 \mathrm{e}-08$ \\
10 & -0.2632012891819914 & $6.45 \mathrm{e}-13$ & $3.164449449931055 \mathrm{e}-02$ & $1.74 \mathrm{e}-13$ \\
15 & -0.2632012891827369 & $7.10 \mathrm{e}-15$ & $3.164449449950190 \mathrm{e}-02$ & $1.67 \mathrm{e}-16$ \\
\hline
\end{tabular}

We conclude the section by testing the approach on two delay systems arising in applications. 

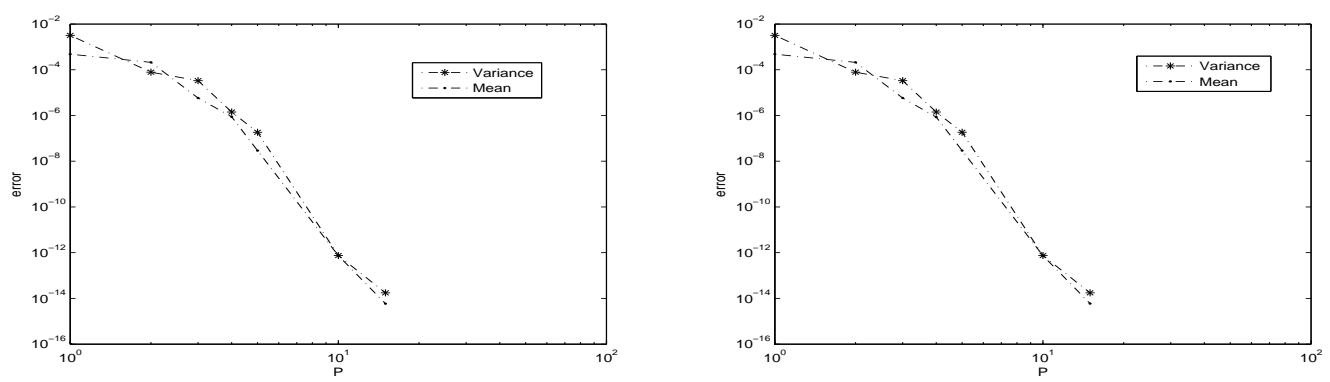

Figure 3. Errors for $U D D E$ (48) (left), $U D D E$ (50) (right) using $N=20$.

5.1. Intravenous glucose tolerance test. Let us consider the DDE modeling the intravenous glucose tolerance test for the dynamics of glucose $G$ and insulin $I$ [26]:

$$
\left\{\begin{array}{l}
G^{\prime}(t)=-b_{1} G(t)-b_{4} G(t) I(t)+b_{7} \\
I^{\prime}(t)=-b_{2} I(t)+\frac{b_{6}}{\tau} \int_{-\tau}^{0} G(t+s) d s
\end{array}\right.
$$

Let us assume that $\tau$ and $b_{6}$ are uncertain. In order to study the effect on the stability of the equilibrium $(\hat{G}, \hat{I})$, we linearize the equation at $(\hat{G}, \hat{I})$ and thus obtain the following linear UDDE of stochastic dimension $M=2$ :

$$
\left\{\begin{array}{l}
\frac{d}{d t} y(t, \xi)=-\left(b_{1}+b_{4} \hat{I}\left(\xi_{2}\right)\right) y(t, \xi)-\left(b_{4} \hat{G}\left(\xi_{2}\right)\right) z(t, \xi), \\
\frac{d}{d t} z(t, \xi)=-b_{2} z(t, \xi)+\frac{b_{6}\left(\xi_{2}\right)}{\tau\left(\xi_{1}\right)} \int_{-\tau\left(\xi_{1}\right)}^{0} y(t+s, \xi) d s,
\end{array}\right.
$$

where $\xi=\left(\xi_{1}, \xi_{2}\right)$. Let $b_{1}=0.0226 e+05, b_{2}=0.0437 e+05, b_{4}=3.80 e-03$, and $\tau\left(\xi_{1}\right)=$ $14.9+6.2 \xi_{1}, b_{6}\left(\xi_{2}\right)=0.074 e+05+0.052 e+05 \xi_{2}$, with $\xi_{1}, \xi_{2}, \sim \mathcal{U}[-1,1]$. The numerical results given in Table 6 show a spectral convergence rate w.r.t. $P$ of the errors computed w.r.t. $E_{N, 40}=$ 7.296148024211029e $-02, V_{N, 40}=6.955156074497970 e-03$. Moreover $\operatorname{Prob}\left\{S_{N, 40}<0\right\}=$ 0.209. By assuming that $b_{4}\left(\xi_{3}\right)=0.0437 e+05+0.208 e+05 \xi_{3}$, with $\xi_{3} \sim \mathcal{U}[-1,1]$, the resulting UDDE has stochastic dimension $M=3$. In this case we get $E_{N, 40}=0.8186, V_{N, 40}=0.888$, and $\operatorname{Prob}\left\{S_{N, 40}<0\right\}=0.426$.

Table 6

Numerical results for the UDDE (52) using $N=20$.

\begin{tabular}{|c|c|c|c|c|}
\hline$P$ & $E_{N, P}$ & $\left|E_{N, P}-E_{N, 40}\right|$ & $V_{N, P}$ & $\left|V_{N, P}-V_{N, 40}\right|$ \\
\hline 5 & $7.29735194437 \mathrm{e}-02$ & $1.20 \mathrm{e}-05$ & $6.948428401299340 \mathrm{e}-03$ & $6.73 \mathrm{e}-06$ \\
10 & $7.29614955045 \mathrm{e}-02$ & $1.52 \mathrm{e}-08$ & $6.955152555470955 \mathrm{e}-03$ & $3.52 \mathrm{e}-09$ \\
20 & $7.29614802422 \mathrm{e}-02$ & $1.00 \mathrm{e}-13$ & $6.955156074479580 \mathrm{e}-03$ & $1.84 \mathrm{e}-14$ \\
\hline
\end{tabular}

5.2. Heat exchanger with control. The temperature dynamics of two fluids $T_{A}, T_{B}$ in a heat exchanger with control can be described by the linear DDEs

$$
\left\{\begin{array}{l}
T_{A}^{\prime}(t)=-(\alpha+\gamma) T_{A}(t)+\alpha T_{B}(t)-\gamma k T_{B}(t-\tau) \\
T_{B}^{\prime}(t)=\beta T_{A}(t)-(\beta+\delta) T_{B}(t)+\delta T_{b}
\end{array}\right.
$$




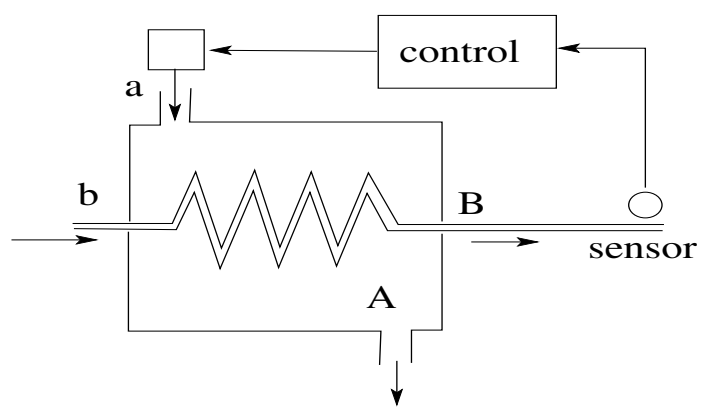

Figure 4. Heat exchanger.

where $\alpha=\frac{1}{C_{A} R_{A B}}, \beta=\frac{1}{C_{B} R_{A B}}, \gamma=\frac{m_{a} c_{a}}{C_{A}}, \delta=\frac{m_{b} c_{b}}{C_{B}}$, and $m_{a}, m_{b}$ are, respectively, the mass flow rate of the hot and the cold fluid entering the device; $c_{a}, c_{b}$ are their specific heats; and $C_{A}, C_{B}, R_{a b}$, and $k$ are suitable constants. $T_{b}$ is the temperature of the cold fluid which represents disturbance [10]. The delay $\tau$ is $\frac{\theta c_{b}}{C_{B} \delta}$ with $\theta$ a positive constant.

The introduction uncertain parameters $\gamma, \delta$ and linearization around equilibrium give the following linear UDDE of stochastic dimension $M=2$ :

$$
\left\{\begin{array}{l}
\frac{d}{d t} y(t, \xi)=-\left(\alpha+\gamma\left(\xi_{1}\right)\right) y(t, \xi)+\alpha z(t, \xi)-\gamma k z\left(t-\tau\left(\xi_{2}\right), \xi\right) \\
\frac{d}{d t} z(t, \xi)=\beta y(t, \xi)-\left(\beta+\delta\left(\xi_{2}\right)\right) z(t, \xi),
\end{array}\right.
$$

where $\xi=\left(\xi_{1}, \xi_{2}\right)$. Let $\alpha=5, \beta=3, k=2.8$, and $\gamma\left(\xi_{1}\right)=3+0.2 \xi_{1}, \delta\left(\xi_{2}\right)=1+0.5 \xi_{2}$ with $\xi_{1}, \xi_{2}, \sim \mathcal{U}[-1,1]$. Let us fix $N=40$. Once more the numerical results in Table 7 indicate a spectral convergence rate of the errors computed w.r.t. $E_{40,40}=-9.5317923319357 e-02$ and $V_{40,40}=2.68624339274695 e-02$. Moreover $\operatorname{Prob}\left\{S_{40,40}<0\right\}=6.23 e-01$.

Table 7

Numerical results for the UDDE (53) using $N=40$.

\begin{tabular}{|c|c|c|c|c|}
\hline$P$ & $E_{N, P}$ & $\left|E_{N, P}-E_{N, 40}\right|$ & $V_{N, P}$ & $\left|V_{N, P}-V_{N, 40}\right|$ \\
\hline 5 & $-9.531716525436598 \mathrm{e}-02$ & $7.58 \mathrm{e}-07$ & $2.686348400478623 \mathrm{e}-02$ & $1.05 \mathrm{e}-06$ \\
10 & $-9.531792327475509 \mathrm{e}-02$ & $4.46 \mathrm{e}-11$ & $2.686243393194849 \mathrm{e}-02$ & $4.48 \mathrm{e}-12$ \\
20 & $-9.531792331935225 \mathrm{e}-02$ & $4.75 \mathrm{e}-15$ & $2.686243392746746 \mathrm{e}-02$ & $2.04 \mathrm{e}-15$ \\
\hline
\end{tabular}

The robustness of the system under the effect of uncertainty in parameters $\gamma, \delta$ can be improved by varying parameter $k$ in the control loop, as the results in Table 8 show, where $N=P=40$.

Table 8

Numerical results for (53) varying $k$ using $N=P=40$.

\begin{tabular}{|c|c|c|c|c|c|c|}
\hline$k$ & 2 & 2.2 & 2.4 & 2.6 & 2.8 & 3 \\
\hline $\operatorname{Prob}\left\{S_{N, P}<0\right\}$ & 0.98 & 0.89 & 0.80 & 0.71 & 0.62 & 0.54 \\
\hline
\end{tabular}

6. Conclusions. We have considered linear DDEs with uncertain parameters, and by reformulating them as abstract DDEs we have proved that mean-square stability can be inferred 
from the spectrum of the infinitesimal generator. In order to quantify the uncertainty propagation in the stability indicator, we have combined the pseudospectral differencing approach with the stochastic collocation method. We have tested it on a number of examples, computing the low-order moments of the output, i.e., mean and variance. We have experimentally investigated convergence w.r.t. to $P$, showing that it is computationally efficient and can represent an alternative to the Monte Carlo method for small stochastic dimension $M$. Since an effective implementation of the method was not the main goal of the paper, we focused instead on the theoretical aspects as well as on the extension of the IG approach from deterministic to uncertain DDEs. Computational improvement through the use of sparse and adaptive collocation points, the derivation of alternative stochastic methods to solve random eigenvalue problems, the extension of the approach for sensitivity analysis, as well as challenging delay models are topics for future work. Time-integration methods for UDDEs will also be studied.

Acknowledgments. The author would like to thank Alexandre Ern, CERMICS (France), and Olivier La Maître, LIMSI-CNRS (France), for introducing her to the polynomial chaos theory; Luisa Arlotti, University of Udine (Italy), for the stimulating discussions; and the referees for their valuable suggestions.

\section{REFERENCES}

[1] O. Arino, M. Hbid, And E. Ait Dads, eds., Delay Differential Equations and Applications, NATO Sci. Ser. II Math. Phys. Chem. 205, Springer-Verlag, Dordrecht, 2006.

[2] O. Arino And E. SÁnchez, A theory of linear delay differential equations in infinite dimensional spaces, in Delay Differential Equations and Applications, O. Arino, M. Hbid, and E. Ait Dads, eds., NATO Sci. Ser. II Math. Phys. Chem. 205, Springer-Verlag, Dordrecht, 2006, pp. 285-346.

[3] F. Augustin And P. Rentrop, Stochastic Galerkin techniques for random ordinary differential equations, Numer. Math., 122 (2012), pp. 399-419.

[4] H. T. Banks And J. A. BuRns, Hereditary control problems: Numerical methods based on averaging approximations, SIAM J. Control Optim., 16 (1978), pp. 169-208, https://doi.org/10.1137/0316013.

[5] H. T. BANKS AND F. KAPPEL, Spline approximations for functional differential equations, J. Differential Equations, 34 (1979), pp. 496-522.

[6] A. Batkai and S. Piazzera, Semigroup for Delay Equations, Research Notes in Mathematics 10, A. K. Peters, Ltd., Wellesley, MA, 2005.

[7] A. Bellen And S. MASET, Numerical solution of constant coefficient linear delay differential equations as abstract Cauchy problems, Numer. Math., 84 (2000), pp. 351-374.

[8] A. Bellen and M. Zennaro, Numerical Methods for Delay Differential Equations, Oxford University Press, Oxford, UK, 2003.

[9] B. Bernier AND A. MANitius, On semigroup in $\mathbb{R}^{n} \times L^{p}$ corresponding to differential equations with delays, Canad. J. Math., 5 (1978), pp. 897-914.

[10] F. Blanchini, D. Casagrande, S. Miani, and U. Viaro, Stable LPV realization of the Smith predictor, Internat. J. Systems Sci., 47 (2015), pp. 2393-2401, https://doi.org/10.1080/00207721.2014.998319.

[11] D. BREDA, Solution operator approximation for characteristic roots of delay differential equations, Appl. Numer. Math., 56 (2006), pp. 305-317.

[12] D. BREDA, Nonautonomous delay differential equations in Hilbert spaces and Lyapunov exponents, Differential Integral Equations, 23 (2010), pp. 935-956.

[13] D. Breda, S. Maset, And R. Vermiglio, Pseudospectral differencing methods for characteristic roots of delay differential equations, SIAM J. Sci. Comput., 27 (2006), pp. 482-495, https://doi.org/10.1137/ 030601600.

[14] D. Breda, S. Maset, And R. Vermiglio, An adaptive algorithm for efficient computation of level curves of surfaces, Numer. Algorithms, 52 (2009), pp. 605-628. 
[15] D. Breda, S. Maset, And R. Vermiglio, Pseudospectral approximation of eigenvalues of derivative operators with non-local boundary conditions, Appl. Numer. Math., 56 (2006), pp. 318-331.

[16] D. Breda, M. Iannelli, S. Maset, and R. Vermiglio, Stability analysis of the Gurtin-MacCamy model, SIAM J. Numer. Anal., 46 (2008), pp. 980-995.

[17] D. Breda, S. MAset, And R. Vermiglio, Numerical approximation of characteristic values of partial retarded functional differential equations, Numer. Math., 113 (2009), pp. 181-242.

[18] D. Breda, S. Maset, And R. Vermiglio, Approximation of eigenvalues of evolution operators for linear retarded functional differential equations, SIAM J. Numer. Anal., 50 (2012), pp. 1456-1483, https://doi.org/10.1137/100815505.

[19] D. Breda, S. Maset, and R. Vermiglio, Stability of Linear Differential Equations: A Numerical Approach with MATLAB, SpringerBriefs Electr. Comput. Eng., Springer-Verlag, Cham, 2015.

[20] D. Breda, O. Diekmann, M. Gyllenberg, F. Scarabel, and R. Vermiglio, Pseudospectral discretization of nonlinear delay equations: New prospects for numerical bifurcation analysis, SIAM J. Appl. Dyn. Syst., 15 (2016), pp. 1-23.

[21] J. P. Boyd, Chebyshev and Fourier Spectral Methods, Dover, Mineola, NY, 2001.

[22] R. H. Cameron and W. T. Martin, The orthogonal development of non-linear functionals in series of Fourier-Hermite functionals, Ann. of Math. (2), 48 (1947), pp. 385-392.

[23] C. Canuto, M. Y. Hussani, A. Quarteroni, and T. A. Zang, Spectral Methods in Fluid Dynamics, Springer Series in Computational Physics, Springer-Verlag, Berlin, 1988.

[24] C. Chicone And Y. Latushrin, Evolution Semigroups in Dynamical Systems and Differential Equations, Math. Surveys Monogr. 70, AMS, Providence, 1999.

[25] J. M. Cushing, Integrodifferential Equations and Delay Models in Population Dynamics, Lecture Notes in Biomathematics 20, Springer-Verlag, Berlin, 1977.

[26] A. De Gaetano And O. Arino, Mathematical modelling of the intravenous glucose tolerance test, J. Math. Biol., 40 (2000), pp. 136-168.

[27] M. C. Delfour, State theory of linear hereditary differential systems, J. Math. Anal. Appl., 60 (1977), pp. 8-35.

[28] R. Denk, M. Möller, And C. Tretter, The spectrum of a parametrized partial differential operator occurring in hydrodynamics, J. Lond. Math. Soc. (2), 65 (2002), pp. 483-492.

[29] R. Denk, M. Möller, And C. Tretter, The spectrum of the multiplication operator associated with a family of operators in a Banach space, Oper. Theory Adv. Appl., 162 (2005), pp. 103-116.

[30] O. Diekmann, S. A. van Gils, S. M. Verduyn Lunel, and H. O. Walther, Delay Equations. Functional, Complex and Nonlinear Analysis, Appl. Math. Sci. 110, Springer-Verlag, New York, 1995.

[31] K. J. Engel And R. NAGel, One-Parameter Semigroups for Linear Evolution Equations, Grad. Texts in Math. 194, Springer-Verlag, New York, 1999.

[32] K. Engelborghs, T. Luzyanina, And D. Roose, Numerical bifurcation analysis of delay differential equations using DDE-BIFTOOL, ACM Trans. Math. Software, 28 (2002), pp. 1-21.

[33] P. ERdős AND P. TuRÁN, On interpolation I. Quadrature and mean convergence in the Lagrangeinterpolation, Ann. of Math., 38 (1937), pp. 142-155.

[34] T. Erneux, Applied Delay Differential Equations, Surv. Tutor. Appl. Math. Sci. 3, Springer-Verlag, New York, 2009

[35] O. G. Ernst, A. Muglera, H.-J. Starkloff, and E. Ullmanna, On the convergence of generalized polynomial chaos expansions, ESAIM Math. Model. Numer. Anal., 46 (2012), pp. 317-339.

[36] D. Ghosh And R. Ghanem, Efficient characterization of the random eigenvalue problem in a polynomial chaos decomposition, Internat. J. Numer. Methods Engrg., 72 (2007), pp. 486-504.

[37] J. K. Hale, Theory of Functional Differential Equations, 2nd ed., Appl. Math. Sci. 3, Springer-Verlag, New York, 1977.

[38] J. K. Hale and S. M. Verduyn Lunel, Introduction to Functional Differential Equations, Appl. Math. Sci. 99, Springer-Verlag, New York, 1993.

[39] T. Insperger and G. StÉPÁn, Semi-discretization for Time-Delay Systems: Stability and Engineering Applications, Appl. Math. Sci. 178, Springer-Verlag, New York, 2011.

[40] E. Isaacson and H. B. Keller, Analysis of Numerical Methods, John Wiley \& Sons, New York, 1966.

[41] K. Іто, On the approximation of eigenvalues associated with functional differential equations, J. Differential Equations, 60 (1985), pp. 285-300. 
[42] K. Iто, Legendre-tau approximation for functional differential equations, in Distributed Parameter Systems, Lect. Notes Control Inf. Sci. 75, F. Kappel, K. Kunisch, and W. Schappacher, eds., SpringerVerlag, Heidelberg, 1985, pp. 191-212.

[43] K. Ito And F. KAPPEL, A uniformly differentiable approximation scheme for delay systems using splines, Appl. Math. Optim., 23 (1991), pp. 217-262.

[44] K. Ito AND F. KAPPEL, Two families of approximation schemes for delay systems, Results Math., 21 (1992), pp. 93-137.

[45] K. Ito And F. Kappel, Evolution Equations and Approximations, Ser. Adv. Math. Appl. Sci. 61, World Scientific, River Edge, NJ, 2002.

[46] K. Ito And R. Teglas, Legendre-tau approximation for functional differential equations, SIAM J. Control Optim., 24 (1986), pp. 737-759, https://doi.org/10.1137/0324046.

[47] F. KAPPEL AND D. SAlamon, On the stability properties of spline approximations for retarded systems, SIAM J. Control Optim., 27 (1989), pp. 407-431, https://doi.org/10.1137/0327021.

[48] V. B. Kolmanovskin And A. Myshkis, Applied Theory of Functional Differential Equations, Mathematics and Its Applications (Soviet Series) 85, Kluwer, Dordrecht, 1992.

[49] V. B. Kolmanovskit and V. R. Nosov, Stability of Functional Differential Equations, Math. Sci. Eng. 180, Academic Press, London, 1986.

[50] Y. Kuang, Delay Differential Equations with Applications in Population Dynamics, Math. Sci. Eng. 191, Academic Press, Boston, MA, 1993.

[51] I. LASIECKA AND A. MANITIUs, Differentiability and convergence rates of approximating semigroups for retarded functional differential equations, SIAM J. Numer. Anal., 25 (1988), pp. 883-907, https://doi. org/10.1137/0725050.

[52] O. Le Maître And O. KNio, Spectral Methods for Uncertainty Quantification with Applications to Computational Fluid Dynamics, Springer-Verlag, New York, 2010.

[53] W. Michiels and S. I. Niculescu, eds., Stability, Control, and Computation for Time-Delay Systems: An Eigenvalue Based Approach, 2nd ed., Adv. Des. Control 12, SIAM, Philadelphia, 2014, https: //doi.org/10.1137/1.9781611973631.

[54] I. G. Rosen, A discrete approximation framework for hereditary systems, J. Differential Equations, 40 (1981), pp. 377-449.

[55] W. PleŚnIAK, Multivariate Jackson inequality, J. Comput. Appl. Math., 233 (2009) pp. 815-820.

[56] D. Salamon, Structure and stability of finite dimensional approximations for functional differential equations, SIAM J. Control Optim., 23 (1985), pp. 928-951, https://doi.org/10.1137/0323053.

[57] D. Xiu AND G. Karniadakis, The Wiener-Askey polynomial chaos for stochastic differential equations, SIAM J. Sci. Comput., 24 (2002), pp. 619-644, https://doi.org/10.1137/S1064827501387826.

[58] D. Xiu And J. S. Hesthaven, High order collocation methods for differential equations with random inputs, SIAM J. Sci. Comput., 27 (2005), pp. 1118-1139, https://doi.org/10.1137/040615201.

[59] D. XIU, Numerical Methods for Stochastic Computation, Princeton University Press, Princeton, NJ, 2010.

[60] W. Sмiтн Erdös-Turán mean convergence theorem for Lagrange interpolation at Lobatto points, Bull. Aust. Math. Soc., 34 (1986), pp. 375-381.

[61] G. StÉpÁn, Retarded Dynamical Systems, Longman, Harlow, UK, 1989.

[62] G. WebB, Functional Differential Equations and Nonlinear Semigroup in $L^{p}$ space, J. Differential Equations, 20 (1976), pp. 71-89.

[63] N. Wiener, The homogeneous chaos, Amer. J. Math., 60 (1938), pp. 897-936.

[64] Z. Wu, AND W. MichiELs, Reliably computing all characteristic roots of delay differential equations in a given right half plane using a spectral method, J. Comput. Appl. Math., 236 (2012), pp. 2499-2514. 